\title{
THE BULGE RADIAL VELOCITY ASSAY (BRAVA). II. COMPLETE SAMPLE AND DATA RELEASE
}

\author{
Andrea Kunder ${ }^{1}$, Andreas Koch ${ }^{2}$, R. Michael Rich ${ }^{3}$, Roberto de Propris ${ }^{1}$, Christian D. Howard ${ }^{4}$, Scott A. Stubbs ${ }^{1}$, \\ Christian I. Johnson ${ }^{3,14}$, Juntai Shen ${ }^{5}$, Yougang Wang ${ }^{6}$, Annie C. Robin ${ }^{7}$, John Kormendy ${ }^{8}$, Mario Soto ${ }^{9}$, \\ Peter Frinchaboy ${ }^{10}$, David B. Reitzel ${ }^{3,11}$, HongSheng ZhaO ${ }^{12}$, AND Livia Origlia ${ }^{13}$ \\ ${ }^{1}$ Cerro Tololo Inter-American Observatory, Casilla 603, La Serena, Chile; akunder@ctio.noao.edu \\ ${ }^{2}$ Zentrum für Astronomie der Universität Heidelberg, Landessternwarte, Königstuhl 12, Heidelberg, Germany \\ ${ }^{3}$ Department of Physics and Astronomy, University of California, Los Angeles, CA 90095-1562, USA \\ ${ }^{4}$ NASA Ames Research Center, MS 211-3 Moffett Field, CA 94035, USA \\ ${ }^{5}$ Key Laboratory for Research in Galaxies and Cosmology, Shanghai Astronomical Observatory, Chinese Academy of Sciences, Shanghai 200030, China \\ ${ }^{6}$ Key Laboratory of Optical Astronomy, National Astronomical Observatoires, Chinese Academy of Sciences, Beijing 100012, China \\ ${ }^{7}$ Observatoire des sciences de l'Univers de Besançon, Besançon, France \\ ${ }^{8}$ Department of Astronomy, The University of Texas at Austin, Austin, TX 78712, USA \\ ${ }^{9}$ Departamento de Física, Universidad de la Serena, Benavente 980, La Serena, Chile \\ ${ }^{10}$ Department of Physics and Astronomy, Texas Christian University, Fort Worth, TX 76129, USA \\ ${ }^{11}$ Griffith Observatory, 2800 East Observatory Road, Los Angeles, CA 90027, USA \\ 12 SUPA, School of Physics and Astronomy, University of St. Andrews, KY16 9SS, UK \\ ${ }^{13}$ INAF, Osservatorio Astronomico di Bologna, Via Ranzani 1, IT 40127 Bologna, Italy \\ Received 2011 August 11; accepted 2011 December 8; published 2012 February 6
}

\begin{abstract}
We present new radial velocity measurements from the Bulge Radial Velocity Assay, a large-scale spectroscopic survey of M-type giants in the Galactic bulge/bar region. The sample of $\sim 4500$ new radial velocities, mostly in the region $-10^{\circ}<l<+10^{\circ}$ and $b \approx-6^{\circ}$, more than doubles the existent published data set. Our new data extend our rotation curve and velocity dispersion profile to $+20^{\circ}$, which is $\sim 2.8 \mathrm{kpc}$ from the Galactic center. The new data confirm the cylindrical rotation observed at $-6^{\circ}$ and $-8^{\circ}$ and are an excellent fit to the Shen et al. $N$-body bar model. We measure the strength of the $\mathrm{TiO} \varepsilon$ molecular band as a first step toward a metallicity ranking of the stellar sample, from which we confirm the presence of a vertical abundance gradient. Our survey finds no strong evidence of previously unknown kinematic streams. We also publish our complete catalog of radial velocities, photometry, TiO band strengths, and spectra, which is available at the Infrared Science Archive as well as at UCLA.
\end{abstract}

Key words: Galaxy: center - stars: abundances - stars: distances - stars: Population II - surveys

Online-only material: color figures

\section{INTRODUCTION}

Only for stars in the Milky Way is it currently possible to examine both the three-dimensional kinematics and composition of a central bulge/bar population, offering a unique laboratory for the study of galaxy formation and evolution. Up until now, there have been very few optical spectra and radial velocities of bulge stars, published in a catalog form, with the spectra and measurements made publicly available. Here we present a catalog of the Bulge Radial Velocity Assay (BRAVA) that has a sample of $\sim 10,000 \mathrm{M}$ giant stars selected from the red giant branch (RGB) of the Two Micron All Sky Survey (2MASS; Skrutskie et al. 2006). This catalog gives the 2MASS magnitudes and positions, our measured radial velocities, and our spectra. This publication presents our complete sample of low-resolution spectra for the BRAVA survey, which covers approximately $-10^{\circ}<l<+10^{\circ}$ and $-4^{\circ}<b<-8^{\circ}$. We also present a rich new data set at $b=-6^{\circ}$ that complements the first results presented in Howard et al. (2008, hereafter Paper I) and the properties of which conform to our other latitudinal studies at $-4^{\circ}$ and $-8^{\circ}$. We expect that the database will ultimately be useful in constraining the dynamical model of the Galactic bulge and in placing limits on the detection of cold streams from fossil infall events. The 2MASS astrometry is of sufficient quality that

\footnotetext{
${ }^{14}$ National Science Foundation Astronomy and Astrophysics Postdoctoral Fellow.
}

proper motions for our sample will be measurable in the next few years.

Although evidence for a barred potential has long been known (e.g., Liszt \& Burton 1980), the modeling of the $2 \mu \mathrm{m}$ surface brightness distribution in the bulge as a bar (Blitz \& Spergel 1991) established that the stellar distribution of the bulge is best modeled as a bar with the position angle of its major axis as $20^{\circ}-45^{\circ}$. The self-consistent dynamical model of a rapidly rotating bar (Zhao 1996) was a significant advance and a number of models have followed; however, additional progress requires constraining new models with a much larger kinematic data set. The question of exactly how the bar structure formed - presumably via some kind of secular evolution (Kormendy \& Kennicutt 2004) but including rapid star formation, winds, and dissipation — can be better addressed with these new large samples. Additionally, the relationship between the bulge, inner disk, thick disk, and halo may be illuminated further. As the coverage and sample sizes analyzed in the bulge increase, it becomes possible to place meaningful limits on the influence of infalling satellites and other types of merger events. The ultimate goal is to move beyond the characterization of the bar's morphology and orientation to the characterization of the bulge/bar as a stellar system with a unique and complex history.

Early surveys of the bulge (Nassau \& Blanco 1958) immediately revealed a distinguishing characteristic, namely, large numbers of $\mathrm{M}$ giants compared with the giant population in globular clusters and the halo. The breakthrough in achieving a physical understanding of the $\mathrm{M}$ giant population arose from 
the surveys undertaken by Victor and Betty Blanco using the newly commissioned grating/prism on the $4 \mathrm{~m}$ telescope at Cerro Tololo Inter-American Observatory (CTIO; Blanco et al. 1984) - an effort that yielded thousands of photographic lowresolution classification spectra. These studies provided the raw material that fed the first radial velocity study (Mould 1986) and infrared surveys (e.g., Frogel \& Whitford 1987). The cool bulge $\mathrm{M}$ giants could be easily identified and exploited because of their brightness in the near-IR (where extinction is low) and by their strong molecular (chiefly titanium oxide [TiO]) bands. Sharples et al. (1990) used an early fiber spectrograph on the Anglo-Australian Telescope to obtain $\sim 300$ spectra of bulge $M$ giants in Baade's Window. However, no study specifically exploited the $\mathrm{M}$ giants as kinematic probes over the entire bulge, even though they could be easily identified by their extremely red $V-I$ colors.

Following the self-consistent, rapidly rotating bar model of Zhao (1996), two efforts modeled newly available, substantial data sets. Sevenster et al. (1999) employed the OH/IR star population to constrain a bar model while the Beaulieu et al. (2000) study used planetary nebulae (PNe) as kinematic probes of the bulge and fit a range of bar models to the data. Both probes offered an advantage in that they are ubiquitous and easy to identify over the whole of the bulge. In the case of the PNe, the numbers are relatively few (373). Unfortunately, PNe are not specific to a given stellar population so disk contamination is a possibility and the total number in the PNe sample is modest. However, Beaulieu et al. (2000) were able to test both the selfconsistent and $N$-body bar models against the PNe kinematic data. Sevenster et al. (1999) were able to draw significant constraints from the $\mathrm{OH} / \mathrm{IR}$ star kinematics, finding a barshaped bulge at a roughly $45^{\circ}$ bar angle. The $\mathrm{OH} / \mathrm{IR}$ stars are also rare, with numbers similar to those of the PNe.

With the release of the 2MASS database, it became possible to easily select $M$ giants over the entirety of the Galactic bulge. The 2MASS survey offered an essentially unlimited supply of kinematic probes. The Bulge Radial Velocity Assay or BRAVA (see also Rich et al. 2007b and Paper I) was conceived to exploit the high quality, uniform photometry and astrometry for this data set. We select red giants from the $K, J-K$ RGB that are approximately at the distance to the Galactic center, $R_{0}=$ $8 \mathrm{kpc}$, and are highly likely to be bulge/bar members. These stars can thus be selected from highly obscured regions of the bulge. The first results of the BRAVA project are given additionally in Rich et al. (2007b), Paper I, Howard et al. (2009), and Shen et al. (2010). The principle result, based on $\sim 4500$ stars, was the confirmation of cylindrical rotation and that the simple bar/boxy bulge model matches the BRAVA kinematics strikingly well with no need for a merger-made classical bulge (Shen et al. 2010). The sample of red giant kinematic probes is now 8585. We also have in progress a new version of the self-consistent model (Y. Wang et al. 2012, in preparation).

As described in Rich et al. (2007b), we used the Hydra spectrograph on the Blanco $4 \mathrm{~m}$ telescope, set at a central wavelength of $\sim 7900 \AA .{ }^{15}$ Many details of the observation and analysis are given in Paper I and will not be repeated here. After some experimentation, we ultimately determined that even with prominent, partially overlapping molecular band absorption, the near-infrared $\mathrm{Ca}$ triplet (CaT) offered the best opportunity to obtain excellent radial velocity cross-correlation.

\footnotetext{
15 The wavelength range for each BRAVA field is given in our data release, as it varies slightly depending on what year the observations were taken.
}

While originally conceived as a purely kinematic study, the data quality of BRAVA allows us, in principle, to investigate the metallicity distribution of the bulge. Only with the addition of chemical abundance information will we ultimately be able to pin down the formation mechanisms that laid the kinematic traces found in our survey. In particular, the presence of radial or vertical metallicity gradients in the bulge may be indicative formation mechanisms (Meléndez et al. 2008; Zoccali et al. 2008; Babusiaux et al. 2010; Johnson et al. 2011) and warrants a well-calibrated, large-number sample, such as BRAVA. Here we report the coordinates, photometry, TiO molecular band strengths, and radial velocities of individual red giants in the BRAVA fields, as well as the mean velocities and velocity dispersions for each field. The data release also includes all of our actual reduced spectra. It is our intention to include proper motion data in the compilation as well. The present paper describes the final data set of BRAVA and presents the BRAVA data release and Web site, which include all available data and spectra from our observing campaign. We also refine the analysis presented in Shen et al. (2010) and confirm that the Galactic bulge appears to consist of a massive bar undergoing pure cylindrical rotation and yields strong limits on the presence of a classical bulge.

The structure of the paper is as follows. We present the observations and spectroscopy, especially insofar as these were changed from those used in Paper I and Howard et al. (2009) in Section 1. Velocity calibrations and the main results are discussed in Section 2, where we also introduce our public data release. Our molecular line index measurements are then introduced in Section 3. Section 4 shows the new data fit to the Shen et al. (2010) $N$-body bar model, and we conclude with the data release in Section 5.

\section{NEW OBSERVATIONS AND SPECTROSCOPY}

The observations presented here were taken in 2008 with the Hydra multifiber bench spectrograph at the Cassegrain focus of the Blanco $4 \mathrm{~m}$ telescope at the CTIO. ${ }^{16}$ Nineteen individual bulge fields were observed in 2008 July and 23 in 2008 August. Additionally, three radial velocity standard stars (HD 177017, HD 218541, and HD 146051), which were already used in Paper I, were acquired during these runs as well as observations of the BRAVA calibration field at $(l, b)=(6,-4)$.

Paper I describes the observational setup and sample selection used to obtain radial velocities of the bulge $\mathrm{M}$ giants. Briefly, in 2005 , our central wavelength was $\sim 7600 \AA$, but every year it was adjusted redward in order to observe the calcium triplet as well as the $\mathrm{TiO}$ band at $\sim 7050$. The specific wavelength range for each BRAVA field is listed in our data release. For our 2008 observations, the KPGLD grating was employed, blazed at $8500 \AA$ and with $1 \times 2$ binning. The central wavelength was $\sim 7900 \AA$ and the effective dispersion was $0.88 \AA$ pixel ${ }^{-1}$ with an effective resolving power of $R \sim 4200$. The spectral range of the new data set included all three CaT lines, and three exposures at $600 \mathrm{~s}$ each were obtained for each field. These fields typically allowed for $\sim 106$ fibers to be placed on $\mathrm{M}$ giants and an additional $\sim 20$ fibers to be used for sky subtraction. The signal-to-noise ratio $(\mathrm{S} / \mathrm{N})$ at $\sim 7500 \AA$ ranges from 10 to 80 with median values of $\sim 35-45$. The variations in $S / N$ arise mainly from transparency at the time the data were taken and the field position in the bulge (extinction, crowding). 16 CTIO is operated by AURA, Inc., under contract to the National Science
Foundation. 
Table 1

BRAVA Rotation and Dispersion Results

\begin{tabular}{|c|c|c|c|c|c|c|c|c|c|c|c|}
\hline $\begin{array}{l}\text { Date of Obs } \\
\text { (UT) }\end{array}$ & $\begin{array}{c}\text { R.A. } \\
(\text { J2000.0) }\end{array}$ & $\begin{array}{c}\text { Decl. } \\
(\mathrm{J} 2000.0)\end{array}$ & $\begin{array}{l}\text { gal } l \\
(\operatorname{deg})\end{array}$ & $\begin{array}{l}\text { gal } b \\
\text { (deg) }\end{array}$ & $\begin{array}{c}\text { Target Fibers } \\
\text { in Config }\end{array}$ & $\begin{array}{l}\text { Reliable } \\
\text { Velocities }\end{array}$ & $\begin{array}{c}\left\langle V_{\mathrm{HC}}\right\rangle \\
\left(\mathrm{km} \mathrm{s}^{-1}\right)\end{array}$ & $\begin{array}{c}\left\langle V_{\mathrm{GC}}\right\rangle \\
\left(\mathrm{km} \mathrm{s}^{-1}\right)\end{array}$ & $\begin{array}{c}\operatorname{err}_{\langle V\rangle} \\
\left(\mathrm{km} \mathrm{s}^{-1}\right)\end{array}$ & $\begin{array}{c}\sigma \\
\left(\mathrm{km} \mathrm{s}^{-1}\right)\end{array}$ & $\begin{array}{c}\operatorname{err}_{\sigma} \\
\left(\mathrm{km} \mathrm{s}^{-1}\right)\end{array}$ \\
\hline 2008 Jul 8 & $17: 57: 05.75$ & $-40: 43: 23.4$ & -9.0 & -8.0 & 108 & 104 & -56.10 & -84.26 & 8.32 & 84.86 & 5.88 \\
\hline 2008 Jul 8 & 18:02:09.79 & $-38: 59: 18.5$ & -7.0 & -8.0 & 102 & 102 & -34.14 & -54.39 & 7.49 & 75.60 & 5.29 \\
\hline 2008 Jul 8 & $18: 14: 40.35$ & $-25: 42: 48.7$ & 6.0 & -4.0 & 107 & 106 & 43.38 & 76.03 & 8.56 & 88.12 & 6.05 \\
\hline 2008 Jul 8 & $18: 36: 28.77$ & $-24: 54: 15.4$ & 9.0 & -8.0 & 109 & 108 & 11.27 & 54.85 & 6.95 & 72.20 & 4.91 \\
\hline 2008 Jul 9 & 18:06:51.74 & $-37: 15: 44.5$ & -5.0 & -8.0 & 110 & 108 & -43.43 & -55.69 & 7.76 & 80.62 & 5.49 \\
\hline 2008 Jul 9 & $18: 11: 25.87$ & $-35: 30: 15.1$ & -3.0 & -8.0 & 115 & 114 & -36.62 & -40.84 & 7.89 & 84.27 & 5.58 \\
\hline 2008 Jul 9 & $18: 22: 18.76$ & $-31: 05: 47.0$ & 2.0 & -8.0 & 105 & 103 & -8.65 & 7.16 & 7.61 & 77.21 & 5.38 \\
\hline 2008 Jul 9 & $18: 24: 32.95$ & $-30: 12: 46.9$ & 3.0 & -8.0 & 109 & 108 & -12.17 & 7.74 & 8.70 & 90.39 & 6.15 \\
\hline 2008 Jul 9 & $18: 28: 19.59$ & $-28: 29: 36.9$ & 5.0 & -8.0 & 106 & 102 & 35.93 & 63.48 & 9.04 & 91.34 & 6.39 \\
\hline 2008 Jul 10 & $17: 41: 55.43$ & $-36: 03: 35.9$ & -6.5 & -3.1 & 65 & 63 & -40.86 & -58.03 & 11.46 & 90.96 & 8.10 \\
\hline 2008 Jul 10 & $17: 50: 54.07$ & $-37: 02: 24.9$ & -6.5 & -5.1 & 89 & 79 & -33.10 & -50.23 & 9.12 & 81.09 & 6.45 \\
\hline 2008 Jul 10 & 18:10:07.40 & $-25: 39: 30.9$ & 5.5 & -3.1 & 88 & 86 & 26.54 & 57.80 & 8.95 & 83.02 & 6.33 \\
\hline 2008 Jul 10 & $18: 32: 36.91$ & $-26: 39: 15.7$ & 7.0 & -8.0 & 104 & 102 & 11.58 & 47.41 & 7.55 & 76.21 & 5.34 \\
\hline 2008 Jul 11 & $17: 59: 22.08$ & $-37: 39: 25.0$ & -6.0 & -6.9 & 102 & 97 & -45.49 & -61.92 & 8.10 & 79.79 & 5.73 \\
\hline 2008 Jul 11 & $18: 17: 49.98$ & $-26: 10: 14.0$ & 6.0 & -4.8 & 107 & 106 & 13.82 & 45.92 & 7.83 & 80.56 & 5.53 \\
\hline 2008 Jul 11 & $18: 22: 15.60$ & $-26: 41: 40.5$ & 6.0 & -6.0 & 104 & 102 & 16.35 & 48.22 & 8.32 & 84.03 & 5.88 \\
\hline 2008 Jul 11 & $18: 26: 03.24$ & $-27: 07: 50.2$ & 6.0 & -6.9 & 99 & 98 & 14.94 & 46.70 & 8.46 & 83.78 & 5.98 \\
\hline 2008 Jul 11 & $18: 29: 57.01$ & $-23: 06: 19.2$ & 10.0 & -5.9 & 93 & 88 & 20.85 & 68.56 & 8.72 & 81.54 & 6.17 \\
\hline 2008 Jul 12 & $17: 55: 51.60$ & $-37: 05: 52.7$ & -6.0 & -6.0 & 102 & 96 & -51.06 & -66.83 & 8.70 & 85.28 & 6.15 \\
\hline 2008 Aug 18 & $17: 45: 20.95$ & $-40: 34: 09.7$ & -10.0 & -6.0 & 104 & 103 & -53.70 & -85.72 & 7.10 & 72.09 & 5.02 \\
\hline 2008 Aug 19 & 17:48:04.97 & $-39: 41: 08.2$ & -9.0 & -6.0 & 105 & $103^{\mathrm{a}}$ & -47.46 & -75.29 & 8.21 & 82.91 & 5.81 \\
\hline 2008 Aug 19 & $17: 50: 44.04$ & $-38: 51: 51.5$ & -8.0 & -6.0 & 106 & 105 & -72.36 & -96.35 & 7.27 & 74.54 & 5.14 \\
\hline 2008 Aug 19 & 17:53:05.49 & $-38: 00: 47.4$ & -7.0 & -6.0 & 111 & 103 & -42.71 & -62.86 & 9.71 & 98.50 & 6.86 \\
\hline 2008 Aug 19 & $18: 26: 34.99$ & $-24: 52: 48.8$ & 8.0 & -6.0 & 109 & 107 & 28.44 & 68.56 & 8.21 & 84.88 & 5.80 \\
\hline 2008 Aug 20 & 17:58:08.76 & $-36: 17: 31.2$ & -5.0 & -6.0 & 109 & 108 & -48.01 & -60.06 & 8.43 & 87.60 & 5.96 \\
\hline 2008 Aug 20 & $18: 28: 33.61$ & $-23: 59: 55.1$ & 9.0 & -6.0 & 111 & 111 & 18.41 & 62.45 & 7.93 & 83.55 & 5.61 \\
\hline 2008 Aug 20 & $18: 30: 21.68$ & $-23: 06: 39.7$ & 10.0 & -6.0 & 105 & 104 & 12.49 & 60.42 & 8.61 & 87.81 & 6.09 \\
\hline 2008 Aug 21 & 18:02:53.91 & $-34: 31: 17.8$ & -3.0 & -6.0 & 111 & 109 & -25.85 & -29.81 & 8.33 & 86.94 & 5.89 \\
\hline 2008 Aug 21 & 18:07:17.57 & $-32: 47: 21.0$ & -1.0 & -6.0 & 111 & 110 & -11.74 & -7.74 & 9.26 & 97.15 & 6.55 \\
\hline 2008 Aug 21 & 18:11:48.68 & $-31: 01: 50.7$ & 1.0 & -6.0 & 114 & 112 & -21.54 & -9.42 & 9.16 & 96.93 & 6.48 \\
\hline 2008 Aug 21 & $18: 16: 10.33$ & $-29: 19: 15.1$ & 3.0 & -6.0 & 115 & 114 & 10.17 & 30.18 & 8.98 & 95.91 & 6.35 \\
\hline 2008 Aug 22 & 18:00:21.58 & $-35: 24: 53.8$ & -4.0 & -6.0 & 114 & 112 & -43.53 & -51.61 & 8.35 & 88.41 & 5.91 \\
\hline 2008 Aug 22 & $18: 05: 10.43$ & $-33: 40: 05.1$ & -2.0 & -6.0 & 112 & 109 & -40.98 & -40.96 & 8.55 & 89.29 & 6.05 \\
\hline 2008 Aug 22 & $18: 20: 20.14$ & $-27: 33: 33.3$ & 5.0 & -6.0 & 111 & 109 & 25.20 & 53.17 & 8.46 & 88.38 & 5.99 \\
\hline 2008 Aug 23 & 18:09:46.09 & $-31: 56: 01.1$ & 0.0 & -6.0 & 110 & 109 & -8.53 & -0.42 & 8.70 & 90.82 & 6.15 \\
\hline 2008 Aug 24 & $18: 13: 54.50$ & $-30: 07: 05.2$ & 2.0 & -6.0 & 112 & 110 & 4.04 & 20.30 & 8.31 & 87.11 & 5.87 \\
\hline 2008 Aug 24 & $18: 18: 25.27$ & $-28: 25: 38.5$ & 4.0 & -6.0 & 116 & 113 & 4.99 & 29.13 & 8.18 & 87.00 & 5.79 \\
\hline 2008 Aug 24 & $18: 24: 29.51$ & $-25: 47: 41.7$ & 7.0 & -6.0 & 111 & 111 & 19.66 & 55.65 & 8.36 & 88.10 & 5.91 \\
\hline 2008 Aug 24 & $18: 26: 51.06$ & $-20: 27: 05.7$ & 12.0 & -4.0 & 105 & 104 & 29.39 & 85.59 & 7.84 & 79.94 & 5.54 \\
\hline 2008 Aug 25 & $18: 30: 38.75$ & $-18: 36: 13.3$ & 14.0 & -4.0 & 110 & 110 & 34.63 & 98.83 & 7.65 & 80.26 & 5.41 \\
\hline 2008 Aug 25 & 18:38:04.33 & $-15: 04: 28.9$ & 18.0 & -4.0 & 106 & 103 & 28.39 & 107.73 & 6.09 & 61.77 & 4.30 \\
\hline 2008 Aug 25 & $18: 45: 38.33$ & $-11: 32: 11.6$ & 22.0 & -4.0 & 103 & 101 & 37.81 & 132.19 & 6.36 & 63.91 & 4.50 \\
\hline
\end{tabular}

Note. ${ }^{\text {a }}$ Number of velocities before the $\sigma$-clipping algorithm removed one star in the calculation of the mean velocity and dispersion.

A complete listing of the observed fields is shown in Table 1 and all of the observed fields from Paper I as well as those presented here are shown in Figure 1.

The M giants were selected from the 2MASS catalog; hence uniform and consistent astrometry and photometry is known for all targets. Figure 2 shows the color-magnitude diagram of the targets. Here the reddening values are taken from Schlegel et al. (1998). Our tests showed that application of the Schlegel et al. (1998) values led to a consistent decrease in the width of the RGB in lower latitude fields and gave a good overall consistency between the various fields, which span a wide range in Galactic latitude and longitude. Reddening and the abundance of target stars decreases as a function of galactic latitude, so the fields at $b=-8^{\circ}$ reach to $K \sim 10.1 \mathrm{mag}$ whereas the fields at $b=-4^{\circ}$ and $b=-6^{\circ}$ go to $K \sim 9.5 \mathrm{mag}$. This selection was shown in Paper I and by Howard et al. (2009) to largely avoid the red clump in the bulge as well as the $\mathrm{M}$ giants belonging to the disk.
The data were reduced and the radial velocities were obtained in a similar manner as described in Paper I. After trimming, overscan-, and bias-correcting the spectra, cosmic rays were removed using the Laplacian edge-detection routine, L. A. Cosmic (van Dokkum et al. 2001). The IRAF task dohydra was used for aperture identification and tracing, flat-fielding, wavelength calibration, and sky subtraction. The cross-correlation routine, xcsao, in IRAF was then used to cross-correlate the spectra against our three radial velocity standard stars, obtain the radial velocities, and correct them to the heliocentric rest frame. The radial velocity standard stars were also used to apply the appropriate zero-point shift of the velocities.

To assess the consistency of our velocity results, a star-bystar comparison of stellar velocities was conducted of the field at $(l, b)=(6,-4)$ as well as overlap stars from the field at $(l, b)=(0,-6)$. Figure 3 shows 101 stellar velocities in the field at $(l, b)=(6,-4)$ and the 16 velocities in the field at 


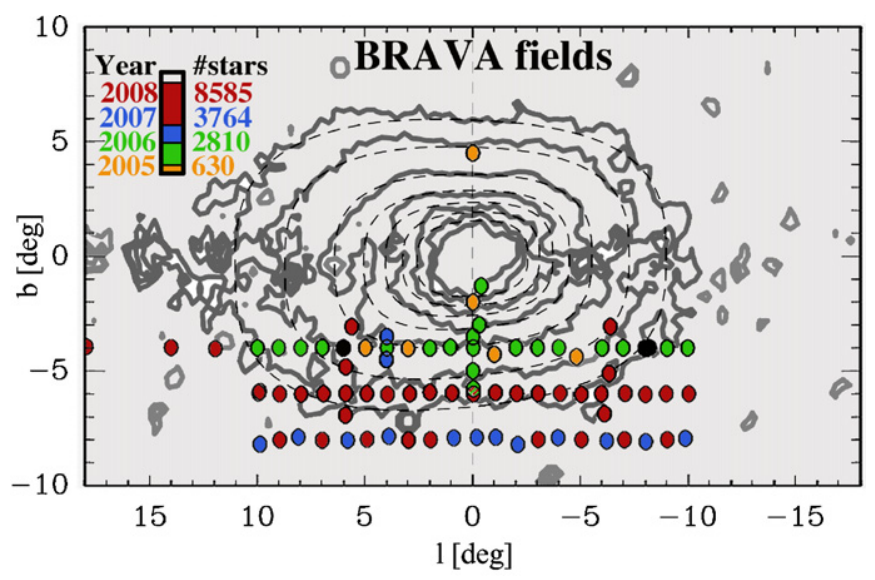

Figure 1. All of the observed BRAVA fields overplotted on the COBE $2 \mu \mathrm{m}$ image (Launhardt et al. 2002). Each field is $40^{\prime}$ and is color-coded to designate the year observed. The black circles are fields observed over multiple years.

(A color version of this figure is available in the online journal.)

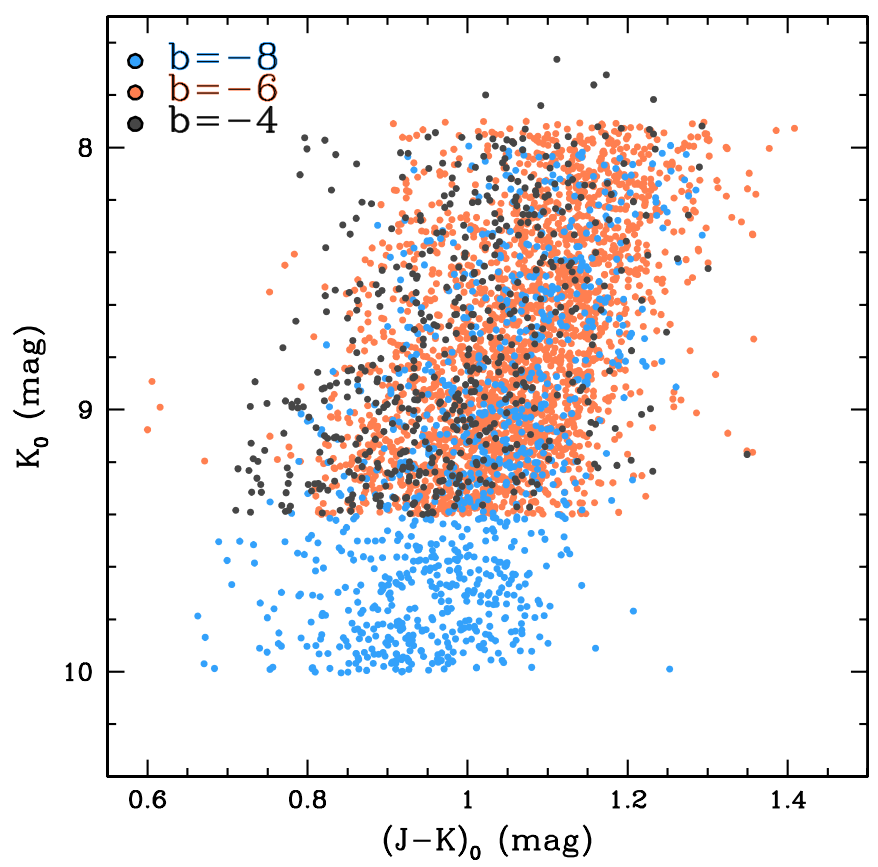

Figure 2. Dereddened 2MASS color-magnitude diagram of the new $4352 \mathrm{M}$ giants for which radial velocities have been determined. Their position on the CMD indicates they are bona fide bulge $\mathrm{M}$ giants.

(A color version of this figure is available in the online journal.)

$(l, b)=(0,-6)$ that are in common between the 2006 and 2008 data. As these velocities agree to within $5 \mathrm{~km} \mathrm{~s}^{-1}$ (the $1 \sigma$ dispersion of the difference), this is the value adopted as the global, individual stellar velocity error. This error is identical to that found for the radial velocities in Paper I. This error is also similar to the velocity precision obtained with our spectra as reported by xcsao.

The heliocentric velocities are corrected for the reflex motion of the Sun following Beaulieu et al. (2000) and the velocity dispersion is given by

$$
\sigma_{\text {intrinsic }}^{2}=\sigma_{\text {observed }}^{2}-\sum_{i=0}^{N}\left[\operatorname{error}^{2}\left(v_{z}\right)\right] /[2(N-1)],
$$

where $\sigma_{\text {observed }}^{2}$ is the observed velocity dispersion of a given field. The second term represents the uncertainties in the

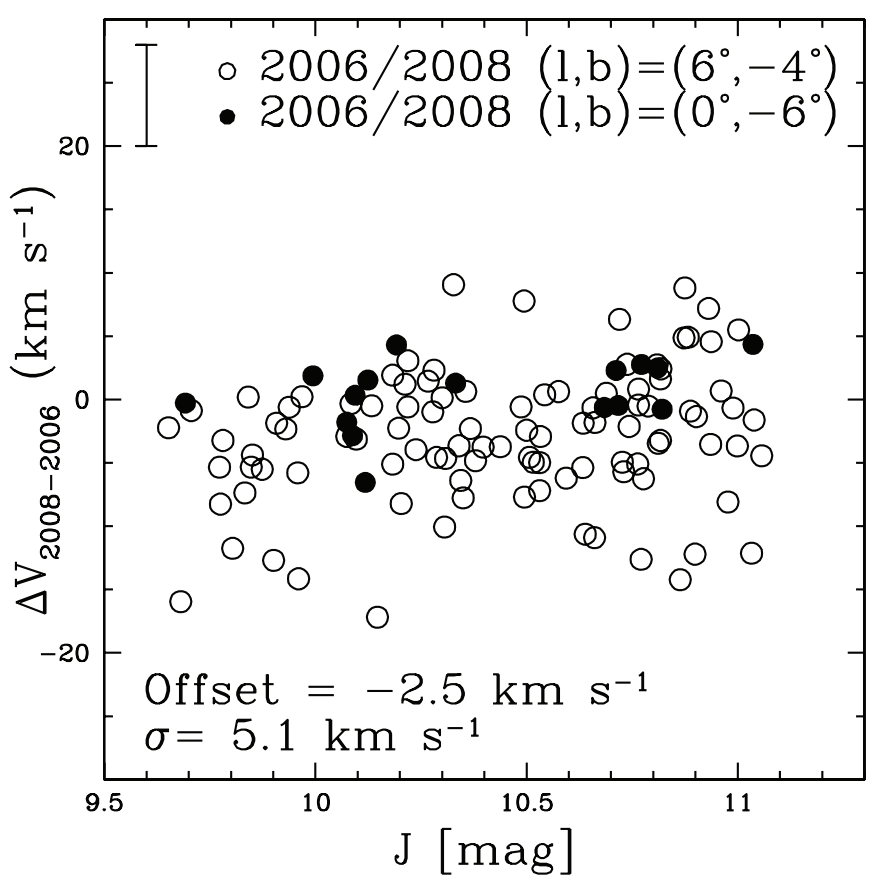

Figure 3. Offset in stellar radial velocities between Paper I observations and the 2008 observations for field $(l, b)=(6,-4)$ (open circles) and for field $(l, b)=(0,-6)$ (closed circles). The size of the errors in the 2008 velocities as reported by xcsao is shown in the top left corner.

individual stellar radial velocity $\left(v_{z}\right)$ measurements, where $N$ is the number of stars in that field. Given the large amount of stars in each field, the second term is negligible, and hence $\sigma_{\text {intrinsic }}^{2}=\sigma_{\text {observed }}^{2}$. The average heliocentric and galactocentric velocities of each field are listed in Table 1 . A $4 \sigma$-clipping algorithm is used to obtain these values; only one star is affected by this clipping.

The complete BRAVA catalog can be accessed at the Infrared Science Archive (IRSA), http://irsa.ipac.caltech.edu/, as well as at the University of California, Los Angeles (UCLA): http://brava.astro.ucla.edu/. Each BRAVA field is designated by its Galactic $l$ and $b$, followed by the average helocentric and galactocentric velocity, error in velocity, dispersion, and error in dispersion, as described above. Lastly the wavelength range of the spectrum is given, as well as the number of stars in each field. The catalog includes all data from 2005, 2006, 2007, and 2008. Following the link beside each field allows the measurements of the individual stars in the field to be obtained, as well as the reduced spectra in fits format. An example of the format for the table of the individual radial velocities is shown in Table 2.

\subsection{Color/Magnitude Bias}

It has been shown in Paper I and Howard et al. (2009) that bulge radial velocities summed over minor- and majoraxis fields consist of a Gaussian distribution with no apparent deviation from a normal distribution. The data analyzed in Paper I are located physically closer to the Galactic center than the new data presented here. As the latter is located closer to the bulge/halo boundary, a comparison between the data presented here and that shown to be from a bona fide RGB bulge sample in Paper I can be used to investigate the possibility of color and/or magnitude bias in the present sample. The bulge RGB stars from the $b=-8^{\circ}$ and $b=-6^{\circ}$ fields are summed and shown in Figure 4. They yield an apparent Gaussian distribution with $\left\langle V_{\mathrm{GC}}\right\rangle=-8 \pm 3 \mathrm{~km} \mathrm{~s}^{-1}$ and $\sigma=96 \pm 2 \mathrm{~km} \mathrm{~s}^{-1}$ for the 
Table 2

Data for Individual Stars in BRAVA Fields

\begin{tabular}{|c|c|c|c|c|c|c|c|c|c|c|c|c|c|c|}
\hline Aperture & $\begin{array}{l}\text { gal } l \\
(\operatorname{deg})\end{array}$ & $\begin{array}{l}\text { gal } b \\
\text { (deg) }\end{array}$ & $\begin{array}{c}\text { R.A. } \\
(\mathrm{J} 2000.0)\end{array}$ & $\begin{array}{c}\text { Decl. } \\
(\mathrm{J} 2000.0)\end{array}$ & $\begin{array}{c}J \\
(\mathrm{mag})\end{array}$ & $\begin{array}{c}H \\
(\mathrm{mag})\end{array}$ & $\begin{array}{c}K \\
(\mathrm{mag})\end{array}$ & $\begin{array}{c}V_{\mathrm{HC}} \\
\left(\mathrm{km} \mathrm{s}^{-1}\right)\end{array}$ & $\begin{array}{c}E \\
(B-V)\end{array}$ & $\begin{array}{c}J_{0} \\
(\mathrm{mag})\end{array}$ & $\begin{array}{c}H_{0} \\
(\mathrm{mag})\end{array}$ & $\begin{array}{c}K_{0} \\
(\mathrm{mag})\end{array}$ & $\begin{array}{c}\mathrm{TiO} \\
(\mathrm{mag})\end{array}$ & 2MASS ID \\
\hline 1 & 9.0256 & -7.8183 & 278.9434 & -24.7927 & 10.640 & 9.940 & 9.724 & -56.4 & 0.405 & 10.275 & 9.707 & 9.575 & -0.0345 & $18354640-2447338$ \\
\hline 3 & 8.6962 & -8.0373 & 279.0005 & -25.1823 & 11.116 & 10.381 & 10.136 & 101.8 & 0.418 & 10.739 & 10.140 & 9.983 & -0.0390 & $18360011-2510562$ \\
\hline 4 & 9.0018 & -7.8323 & 278.9456 & -24.8201 & 10.468 & 9.625 & 9.298 & -84.1 & 0.403 & 10.105 & 9.393 & 9.150 & 0.0055 & $18354694-2449123$ \\
\hline 7 & 9.2062 & -7.7561 & 278.9694 & -24.6047 & 10.643 & 9.843 & 9.590 & -40.8 & 0.382 & 10.298 & 9.623 & 9.450 & -0.0336 & $18355264-2436170$ \\
\hline 8 & 8.9748 & -7.9853 & 279.0837 & -24.9118 & 10.018 & 9.043 & 8.722 & 115.9 & 0.418 & 9.641 & 8.802 & 8.569 & 0.0895 & $18362009-2454424$ \\
\hline
\end{tabular}

Note. Spectra are available at http://brava.astro.ucla.edu/ and http://irsa.ipac.caltech.edu/.

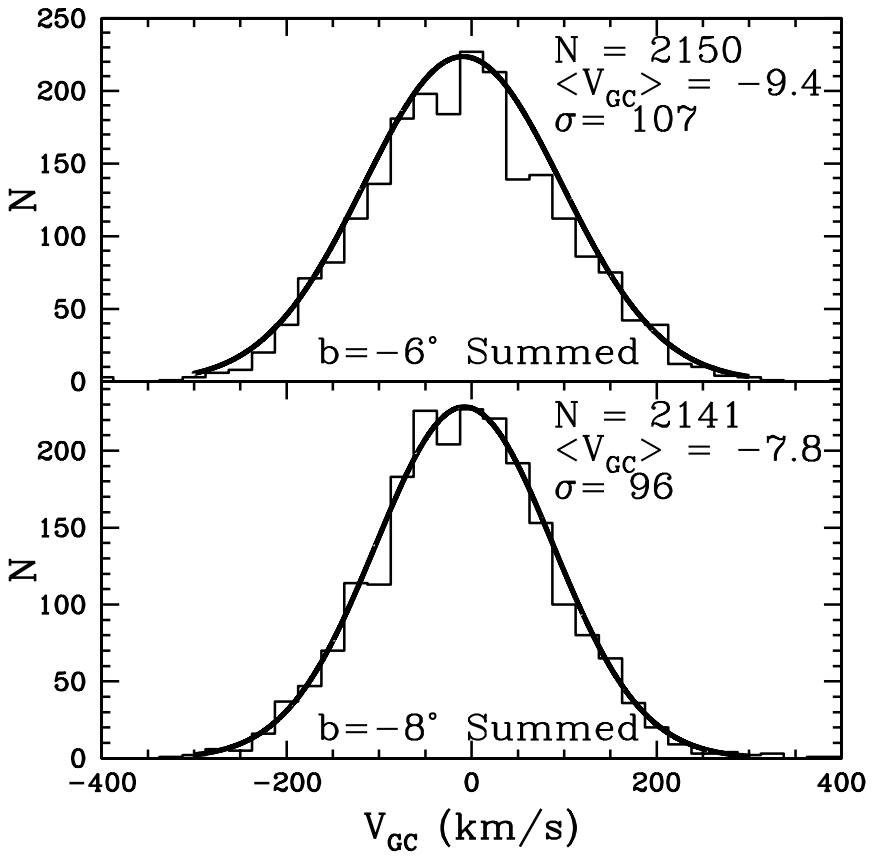

Figure 4. Histogram of all bulge RGB star galactocentric velocities for the fields at $b=-8^{\circ}$ (bottom) and $b=-6^{\circ}$ (top). Both distributions are normally distributed with negligible skew and kurtosis, which is consistent with a single kinematic population.

$b=-8^{\circ}$ fields and $\left\langle V_{\mathrm{GC}}\right\rangle=-10 \pm 3 \mathrm{~km} \mathrm{~s}^{-1}$ and $\sigma=107 \pm$ $3 \mathrm{~km} \mathrm{~s}^{-1}$ for the $b=-6^{\circ}$ fields. Both of these curves are consistent not only with each other, but also with those from Paper I, which suggests that these bulge samples in fact consist of a homogeneous, normally distributed stellar population. Any subpopulation is not significant enough to cause a departure from the Gauassian distribution.

\section{3. $\mathrm{TiO} \varepsilon$ AS METALLICITY INDICATOR}

While our spectral range, in principle, offers a wealth of metallicity indicators, the standard calibrations of the line strengths of the three prominent Ca II lines at 8498, 8542, and $8662 \AA$ onto an $[\mathrm{Fe} / \mathrm{H}]$ scale (e.g., Rutledge et al. 1997) fail for the cool $\mathrm{M}$ giants that make up the majority of the BRAVA sample. Here, the largest problem for measuring the CaT is its contamination with the strong $\mathrm{TiO} \varepsilon$ band at $8430 \AA$, which becomes progressively stronger with decreasing $T_{\text {eff }}$. In fact, this band has a strong dependence on stellar effective temperature (e.g., Milone \& Barbuy 1994). Since we can estimate our $T_{\text {eff }}$ scale using the 2MASS infrared colors (Figure 6), measurements of the band strength then allow us to explore the variations with stellar metallicities (Sharples et al. 1990). In particular, at an

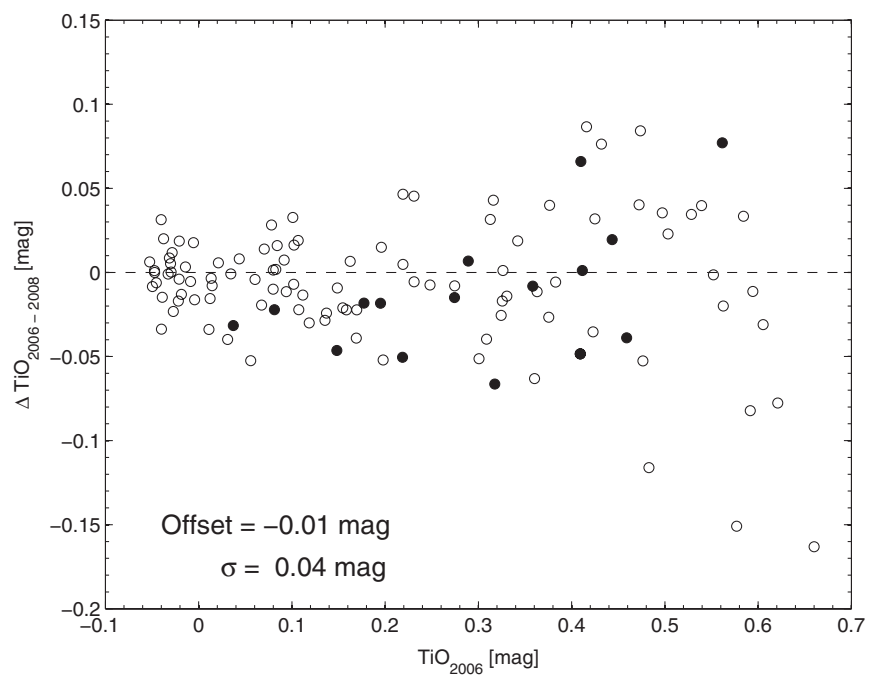

Figure 5. Offset in $\mathrm{TiO} \varepsilon$ between stars observed in both 2006 and 2008. The filled circles correspond to stars in field $(l, b)=(0,-6)$, and the open circles correspond to stars in field $(l, b)=(6,-4)$.

expected mean of $[\mathrm{Fe} / \mathrm{H}] \sim-0.5$ dex, the $\mathrm{TiO} \varepsilon$ band will be very prominent in the metal rich bulge stars.

As a first step toward a comprehensive metallicity distribution, we follow Sharples et al. (1990) in extracting a TiO-strength index, $\mathrm{TiO} \varepsilon$, from our spectra, which is defined as a magnitude via

$$
\mathrm{TiO} \varepsilon=-2.5 \log F_{1} / F_{2},
$$

where the fluxes are obtained from straight integration in the bands from 8370 to $8420 \AA\left(F_{1}\right)$ and 8440 to $8490 \AA\left(F_{2}\right)$. The $1 \sigma$ error on this number, estimated from the variance in those spectral bands, is typically 0.05 mag. Values of each star's TiO index are provided in the final BRAVA catalog. ${ }^{17}$ Note that this molecular band is not measurable in the spectra taken in 2005 due to the instrumental setup.

Figure 5 compares our $\mathrm{TiO}$ measurements for the two sets of overlapping spectra, as is already done for the velocities in Figure 3. The $1 \sigma$ scatter between both sets of $\mathrm{TiO} \varepsilon$ is 0.04 mag with a mean difference below $0.01 \mathrm{mag}$. This is fully consistent with the uncertainty on the index, estimated above from the spectral variance.

In Figure 6 the $\mathrm{TiO} \varepsilon$ index is shown as a function of infrared color. The distribution of the molecular index in our spectra shows a narrow band around zero, i.e., there is no discernible

17 In a future paper (A. Koch et al. 2012, in preparation) we will explore the reliable calibration of the $\mathrm{CaT}$ line strengths onto metallicity for a subsample of the stars with warmer temperatures, in which the CaT is yet unaffected by TiO absorption. Our measured equivalent widths will then be added to this BRAVA release. 


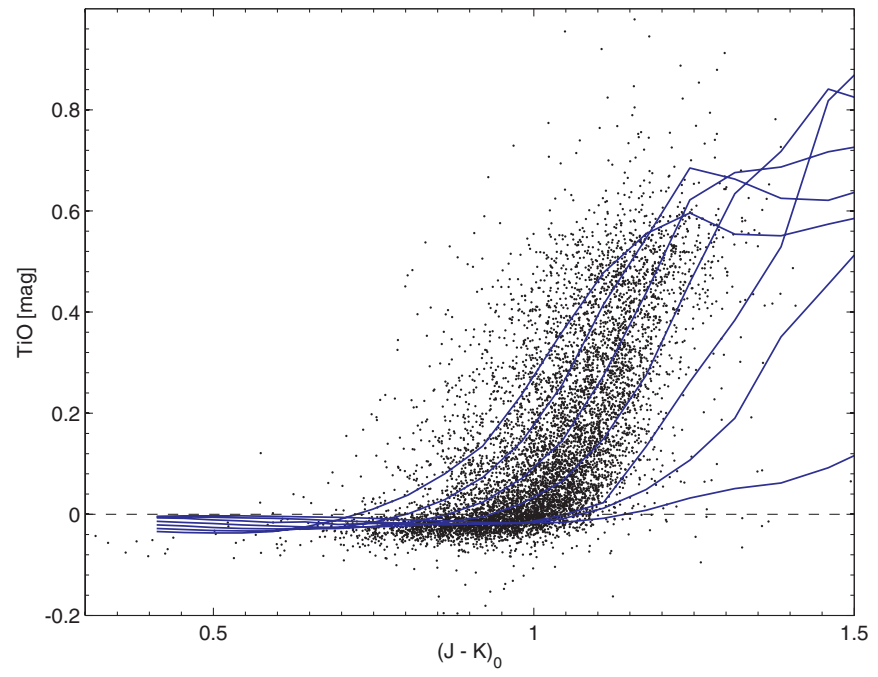

Figure 6. $\mathrm{TiO} \varepsilon$ index (after Sharples et al. 1990) vs. infrared color. The solid lines in the bottom panel are based on $\alpha$-enhanced Phoenix models for metallicities, $[\mathrm{M} / \mathrm{H}]$ between -2.5 and +0.5 dex (right to left) in steps of 0.5 , adopting $\log g=1$. A typical error bar is indicated in the top left corner.

(A color version of this figure is available in the online journal.)

TiO depression, but a well-defined pseudo-continuum in the warmer stars. This holds for a broader range in $J-K$ between 0.7 and 1.1 mag. However, for stars redder than $J-K \gtrsim 1.0$ (corresponding to $T_{\text {eff }} \lesssim 3700 \mathrm{~K}$ ) there is a notable presence of progressively strong bands, leading to a linear rise in the $\mathrm{TiO}$ index.

\subsection{Phoenix Models}

A dispersion in $\mathrm{TiO} \epsilon$ at fixed $(J-K)$ can be due to both differential reddening and metallicity spread. To illustrate this, we consulted a set of synthetic spectra based on the Phoenix stellar atmosphere models. These offer a large grid of stellar atmospheric parameters and a spectral library in high resolution $^{18}$ (Hauschildt et al. 1999, 2003). In particular, its meticulous treatment of cool stellar atmospheres and its vast input sets of molecular opacities makes this synthetic library ideally suited for comparison with the BRAVA data. Our chosen model grid consisted of $T_{\text {eff }}$ ranging from 3200 to $4500 \mathrm{~K}$ in steps of $100 \mathrm{~K}, \log g=0,0.5,1.0,1.5$, metallicities $[\mathrm{M} / \mathrm{H}]=$ -2.5 to +0.5 in increments of $0.5 \mathrm{dex}$, and $\alpha$-enhancement of +0.4 dex as well as solar-scaled abundances, i.e., $[\alpha / \mathrm{Fe}]=0$ dex. This generously comprises the range of parameters spanned by the BRAVA late-type giant sample and is also consistent with the range in spectral types targeted in abundance studies of comparable stellar samples (Rich \& Origlia 2005; Fulbright et al. 2006; Rich et al. 2007a). The synthetic spectra were then smoothed with a Gaussian kernel to mimic the resolution of the BRAVA spectra. Finally, all of the relevant quantities such as the $\mathrm{TiO} \varepsilon$ index were derived from these convolved spectra in the same manner as for the observed data.

As Figure 6 indicates, the bulk of the target stars falls near $[\mathrm{M} / \mathrm{H}]$ of -0.5 dex, which is consistent with the metallicity distribution functions in the literature ${ }^{19}$ (e.g., Rangwala \& Williams 2009; De Propris et al. 2011; Johnson et al. 2011). Moreover, stars are found to cover a broad range from supersolar down to low metallicities around -1.5 dex, with a hint

\footnotetext{
18 ftp://phoenix.hs.uni-hamburg.de/GAIA_HighRes/Spectra/

19 Note, however, that such observations strictly derive MDFs based on iron abundances, $[\mathrm{Fe} / \mathrm{H}]$, and a quantitative comparison with the models' global metallicity may not be straightforward.
}

of a very metal poor extension toward -2 dex. A detailed, quantitative study of the abundance properties of the BRAVA sample will be presented in a forthcoming paper.

A number of points on the blue side of Figure 6 seem inconsistent with the Phoenix models. One reason for this may be that the Schlegel et al. (1998) reddening values overestimate the reddening, and these points should then come from the most reddened fields. We should explore which fields account for these points. When comparing the Schlegel et al. (1998) $E(B-V)$ values for 330 BRAVA stars located within the Gonzalez et al. (2011) reddening map, a $\sim 0.15$ mag offset in $E(B-V)$ is seen, with the Schlegel et al. (1998) values being systematically larger. This translates to $\sim 0.08$ mag in $(J-K)$; hence the Gonzalez et al. (2011) reddening values would move the $(J-K)_{0}$ values in Figure 6 to the red by roughly the spacing between the Phoenix curves, and the number of points on the blue side of the +0.5 dex Phoenix model would be decreased. Another possible contribution of uncertainties in the TiO strength is the uncertainty in the 2MASS photometry, which is roughly 0.03 to $0.1 \mathrm{mag}$ in $(J-K)$. There has also been the long standing problem in the bulge (Frogel \& Whitford 1987) that the bulge giants appear to have $\mathrm{TiO}$ that is much too high for their $(J-K)$. Further surveys such as APOGEE will obtain high-resolution, high $\mathrm{S} / \mathrm{N}$ infrared spectroscopy of red giant stars across the Galactic bulge and may shed light on this issue.

\subsection{Metallicity Gradients}

Recent high-resolution spectroscopic studies of the bulge find a vertical abundance gradient in the sense of lower metallicities toward higher latitudes (e.g., Zoccali et al. 2008; Johnson et al. 2011; Gonzalez et al. 2011). Based on the large BRAVA sample, this issue can also be investigated (although, we only do it qualitatively in this paper). Figure 7 shows the $\mathrm{TiO}$ metallicity indicator separated by Galactic latitude. The Galactic longitude of these fields is $l=0^{\circ}$, and the Phoenix model for a metallicity of -0.5 dex is overplotted to guide the eye. It becomes immediately obvious that there is in fact a systematic difference between the fields with a tendency for the lower latitude fields to have higher metallicities - a trend consistent with recent suggestions of metallicity gradients across the bulge derived from the $\mathrm{K}$ giants (e.g., Zoccali et al. 2008; Johnson et al. 2011). This is also consistent with results of the $\mathrm{M}$ giant population from Frogel et al. (1999); they use IR CMDs to show there is a gradient in mean metallicity along the minor axis in the range $0^{\circ}>b>$ $-10^{\circ}$. Moreover, we find that the most prominent trend concerns the increase in metallicity spread as one approaches the inner bulge regions. However, a detailed analysis of the uncertainties in the reddening and photometry in the inner bulge regions as compared to those in the outer regions is necessary, especially as this is not seen in the high-resolution data (i.e., Zoccali et al. 2008; Johnson et al. 2011).

Finally, we show in Figure 8 the entire sample segregated by stellar ( $K$-band) magnitude. Among all of the magnitude bins the color-TiO distributions are broadly consistent with each other, bolstering our findings from Section 2.1 which argue against any color/magnitude bias in the BRAVA sample, also with regard to metallicity. The only apparent exception is the faintest bin, which exclusively contains stars at the highest latitude (Figure 2). Thus, the metallicity gradient also permeates this (magnitude-) subsample.

If the Schlegel et al. (1998) extinction were higher than that of Gonzalez et al. (2011), it could in principle cause our derived 


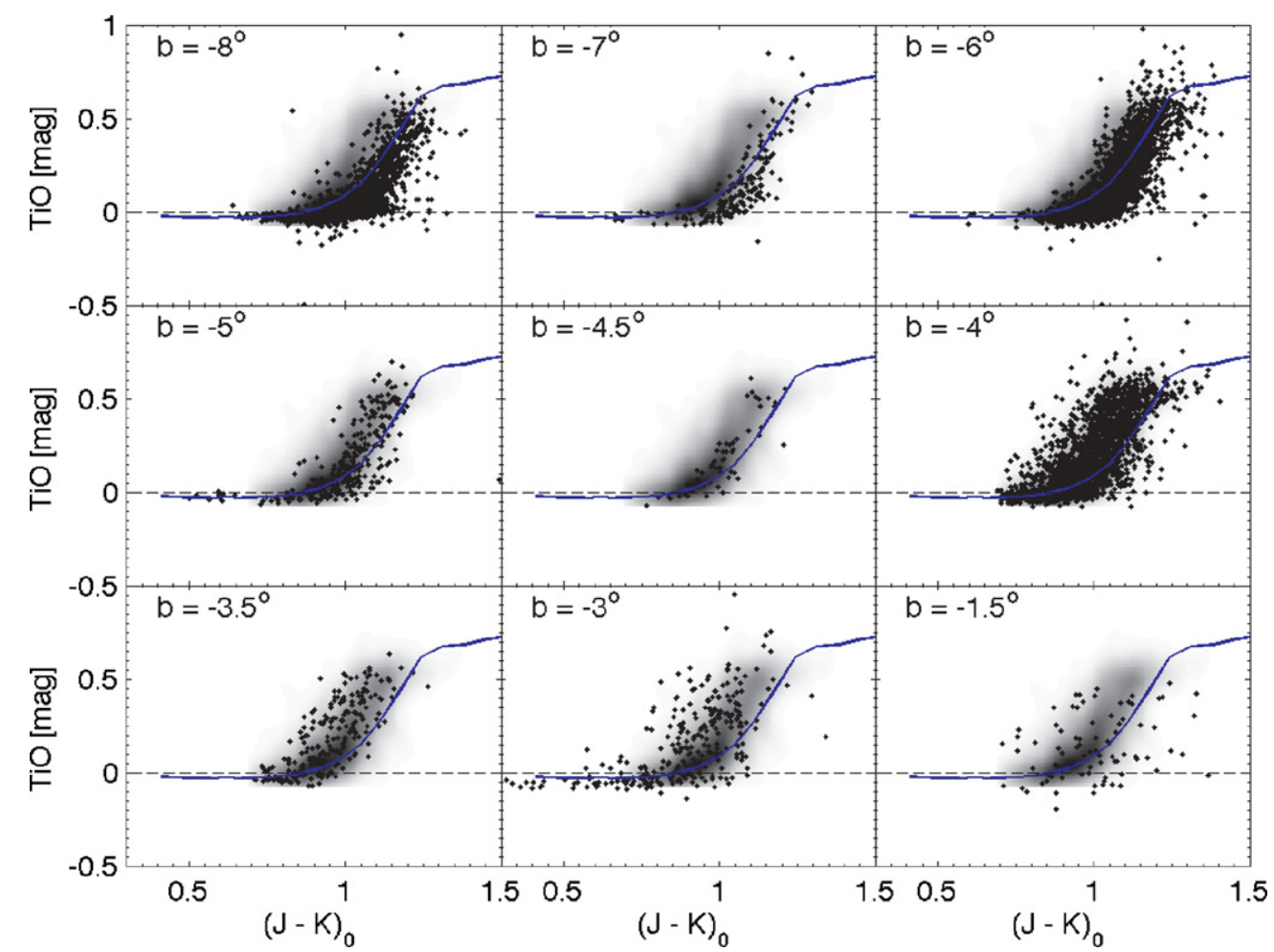

Figure 7. Same as Figure 6, but separated by the Galactic latitudes covered by BRAVA. The gray-shaded region indicates the distribution in the $b=-4^{\circ}$ field and the Phoenix model for a metallicity of $-0.5 \mathrm{dex}$ is overplotted.

(A color version of this figure is available in the online journal.)

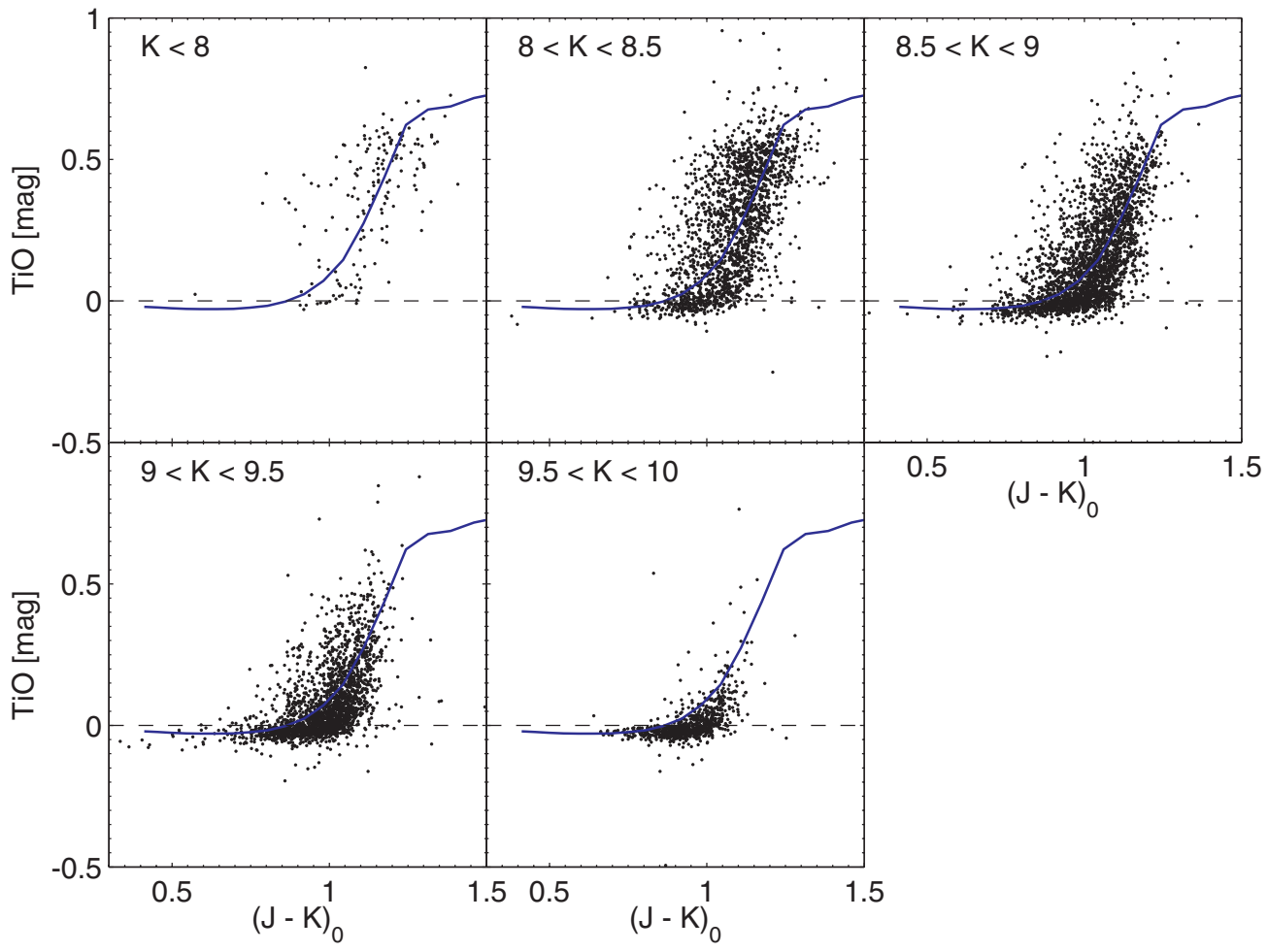

Figure 8. Same as Figure 6, but segregated by stellar magnitude. (A color version of this figure is available in the online journal.)

$(J-K)_{0}$ to be too blue in the fields of greater extinction. As discussed above, the lower $E(B-V)$ of Gonzalez et al. (2011) has an 0.08 mag effect on $(J-K)_{0}$. However, this is unlikely to mimic a metallicity gradient. Small-scale variations in reddening are particularly strong in the inner regions, but these regions are mostly constrained to $|b|<4^{\circ}$ (see Gonzalez et al. 2011, their Figures 6 and 7). The metallicity gradient in the BRAVA data extends all the way to $b=-1.5$, but is also 


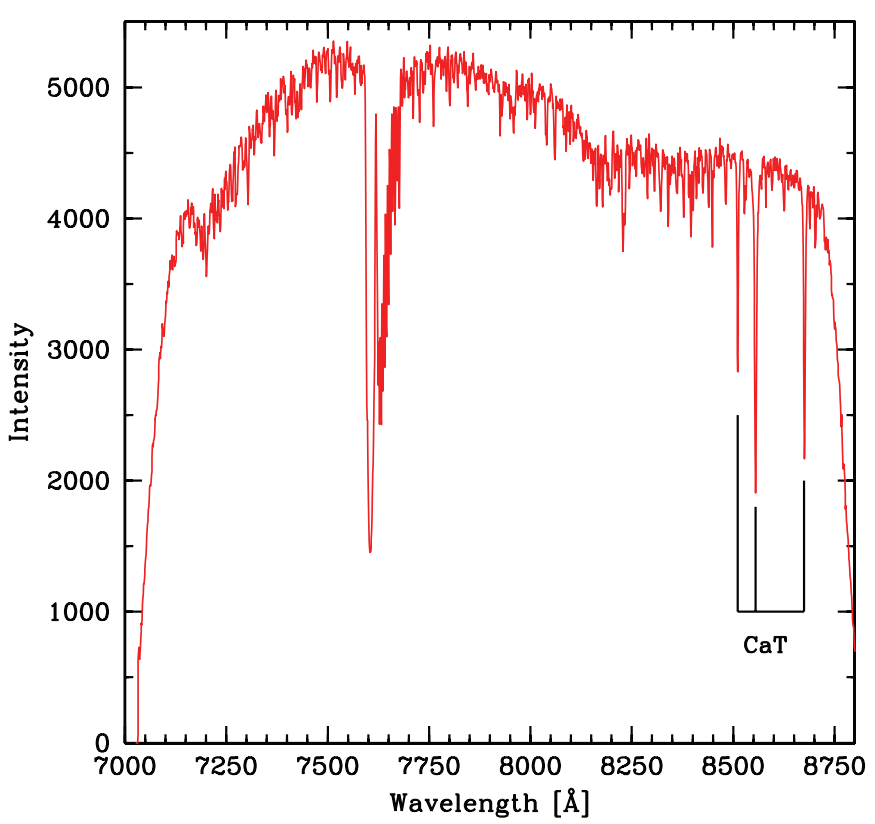

Figure 9. Wavelength-calibrated spectrum of a velocity outlier in our sample with a heliocentric velocity of $447 \mathrm{~km} \mathrm{~s}^{-1}$. The CaT lines are labeled in the spectrum for reference.

(A color version of this figure is available in the online journal.)

prominent when considering the $b=-8^{\circ}$ to $b=-4^{\circ}$ fields. Furthermore, we find a similar scatter $(\sim 0.1 \mathrm{mag})$ and a similar offset $(\sim 0.15 \mathrm{mag})$ in $\Delta E(B-V)$ between Schlegel et al. (1998) and Gonzalez et al. (2011) of our $b=-4^{\circ}$ and $b=-6^{\circ}$ stars. Yet these fields still show a metallicity gradient. This strongly suggests that reddening uncertainties cannot fully explain away the slight metallicity gradient we are seeing.

\section{OUTLIERS}

Radial velocity surveys of the Milky Way halo have the potential to identify rare velocity outliers that result from dynamical processes throughout the Galaxy. The most obvious outlier in our sample is 2MASS 17464606-3937523 with a heliocentric radial velocity of $447 \mathrm{~km} \mathrm{~s}^{-1}$. Its spectrum is shown in Figure 9. The $\mathrm{S} / \mathrm{N}$ is high, and to first order it looks metal poor. Unfortunately, at $(l, b)=(-9.0433,-5.7386)$, it is not in the OGLE-II proper motion catalog (Sumi et al. 2004). Hypervelocity stars (HPVs) were first discovered by Brown et al. (2005) and are generally B-type stars moving 2-3 times the Galactic escape velocity. At a heliocentric velocity of $\sim 450 \mathrm{~km} \mathrm{~s}^{-1}$, the star may not qualify as a true HPV star, although at $r=50$ to $100 \mathrm{kpc}$ unbound stars have such velocities (Kenyon et al. 2008). A preliminary $[\mathrm{Fe} / \mathrm{H}]$ based on the $\mathrm{CaT}$ is $-0.86 \pm 0.05$ dex with an error purely based on the continuum variance and magnitude errors, not accounting for uncertainties in the calibration coefficients. This is not an unusual $[\mathrm{Fe} / \mathrm{H}]$ value for a bulge star.

\section{RESULTS AND ANALYSIS}

Radial velocities, especially in minor axis fields, can constrain bulge models. Figure 10 shows the dispersion profile and rotation curve for the minor-axis strips at $l=-6^{\circ}, l=6^{\circ}$, and $l=0^{\circ}$. The predictions of the Shen et al. (2010) models is overplotted. The Shen model uses a cylindrical particle-mesh code to construct an $N$-body model to the BRAVA data. The initial parameters adopted are an unbarred disk and a rigid

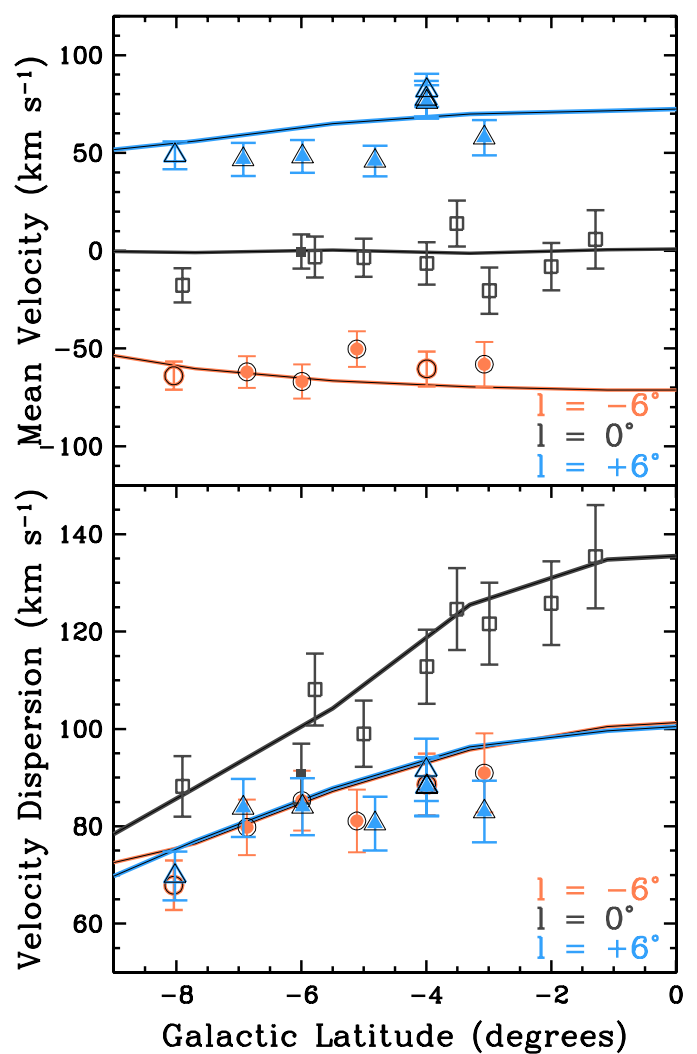

Figure 10. Velocity dispersion profile (bottom) and rotation curve (top) for the $l=-6^{\circ},+6^{\circ}$, and $0^{\circ}$ strips. The open symbols indicate data already published and the filled symbols indicate the data presented here. The model of Shen et al. (2010) is overplotted in good agreement with the observations.

(A color version of this figure is available in the online journal.)

pseudo-isothermal halo potential (see Shen et al. 2010 for details). In this model, the Milky Way self-consistently develops a bar, which buckles and thickens in the vertical directions. Hence, there is no classical bulge component, and the best-fit model predicts a bar half-length of $\sim 4 \mathrm{kpc}$, extending $20^{\circ}$ from the Sun-Galactic-center line. The additional data presented here allow the Shen model to be compared to two more minor axis fields. As seen in Shen et al. (2010), the data agree well with the Shen model for both the mean velocity and velocity dispersion observations.

Figure 11 shows the dispersion profile and rotation curve for the major-axis strips at $b=-4^{\circ}, b=-6^{\circ}$, and $b=-8^{\circ}$. The $b=-4^{\circ}$ data come from Paper I and half of the $b=-8^{\circ}$ data come from Howard et al. (2009). The Shen model matches the observations well, validating the results of Shen et al. (2010). It is also worth noting that the rotation curves at $b=-4^{\circ},-6^{\circ}$, and $-8^{\circ}$ are remarkably similar, suggesting that the Galaxy's bulge rotates cylindrically. This was suggested by Howard et al. (2009), but here the observational data are more plentiful and include additional fields at $b=-6^{\circ}$. Thus our bolstering of the cylindrical rotation signal is further evidence that the bulge is an edge-on bar, as predicted by the Shen model.

The velocity dispersion drops as one moves further in latitude from the Galactic disk plane. A hotter model contributes to a large velocity dispersion and the nucleus-spheroid component of the model is hot. However, the BRAVA data show remarkable agreement with the disk/bar component of the model with a relatively flat dispersion profile at $\sim 70 \mathrm{~km} \mathrm{~s}^{-1}$ contrasting with the spheroid dispersion, which is predicted to be at $\sim 120 \mathrm{~km} \mathrm{~s}^{-1}$. 


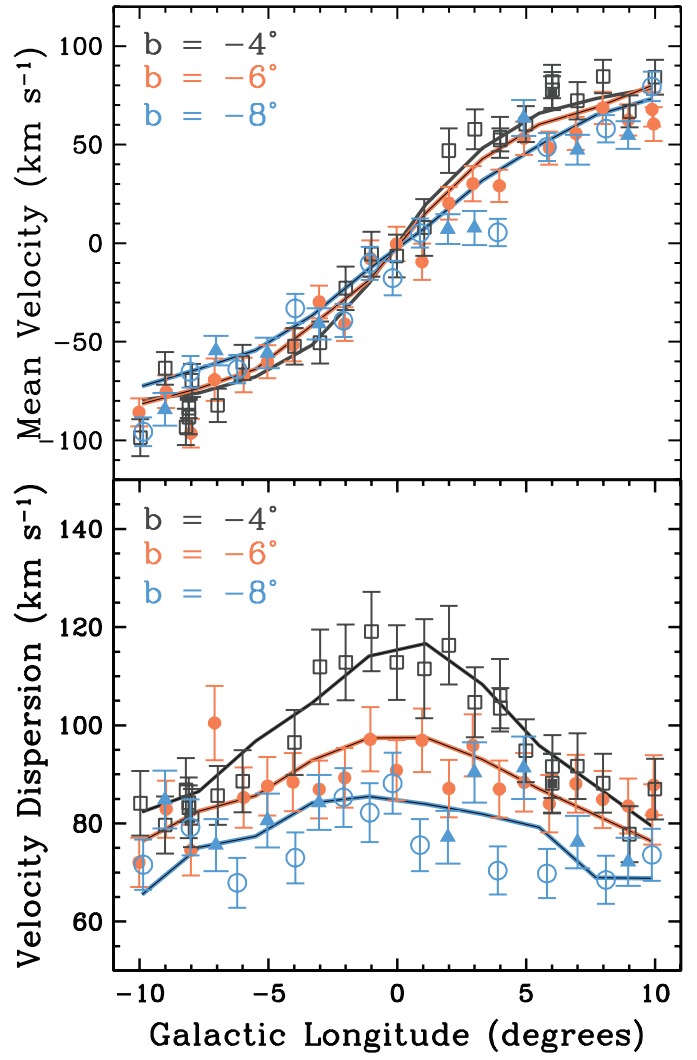

Figure 11. Velocity dispersion profile (bottom) and rotation curve (top) for the $b=-4^{\circ},-6^{\circ}$, and $-8^{\circ}$ strips. The filled symbols indicate data already published and the open symbols indicate the data presented here.

(A color version of this figure is available in the online journal.)

The turnover seen in the rotation curve at $b=-4^{\circ}$ is not evident at $b=-6^{\circ}$ or $b=-8^{\circ}$.

Additionally, in Figure 12, the BRAVA fields extending past the main body of the bulge at $l>10^{\circ}$ are plotted. These observations lie outside the high surface brightness boundary of the COBE bulge and can be used to examine the extent of the bar/bulge and to look for signs of disk contamination. The full length of the bar is thought to be on the order of 3-4 kpc (Hammersley et al. 2000; Bissantz \& Gerhard 2002; CabreraLavers et al. 2007) and since the bar's pattern speed is relatively rapid, it effectively "controls" the region in which it lives. At the distance of the Galactic center $(7.9 \mathrm{kpc}), 4 \mathrm{kpc}$ at the bar angle of $\sim 20$ deg corresponds to $\pm l \sim 16^{\circ}$. Indeed, the fields at $l>15^{\circ}$ show a drop in velocity dispersion as well as a higher mean velocity, which is due presumably to the presence of the inner disk component in these fields. Figure 12 also shows the observations from a sample of $373 \mathrm{PNe}$ (Beaulieu et al. 2000); the BRAVA data confirm the rotation seen by the PNe at $|l|>$ $12^{\circ}$, as well as a drop in the velocity dispersion in these fields. The region covered in the Beaulieu et al. (2000) data is $-20^{\circ}<$ $l<20^{\circ}$ and $-5^{\circ}>b>-10^{\circ}$. Observations at $|l|>12^{\circ}$ place important constraints for Galactic bulge theoretical models and predictions, such as the bar angle (Martinez-Valpuesta 2011, private communication).

The galactocentric velocity distributions of the new bulge fields are now presented in Figure 13. As with Paper I, a bin width of $25 \mathrm{~km} \mathrm{~s}^{-1}$ is used, driven by the approximate dispersion expected for cold components like a dissolving cluster or dwarf galaxy $\left(\sim 10 \mathrm{~km} \mathrm{~s}^{-1}\right)$. Simulations by Reitzel et al. (2007) show that with the number of stars we have in

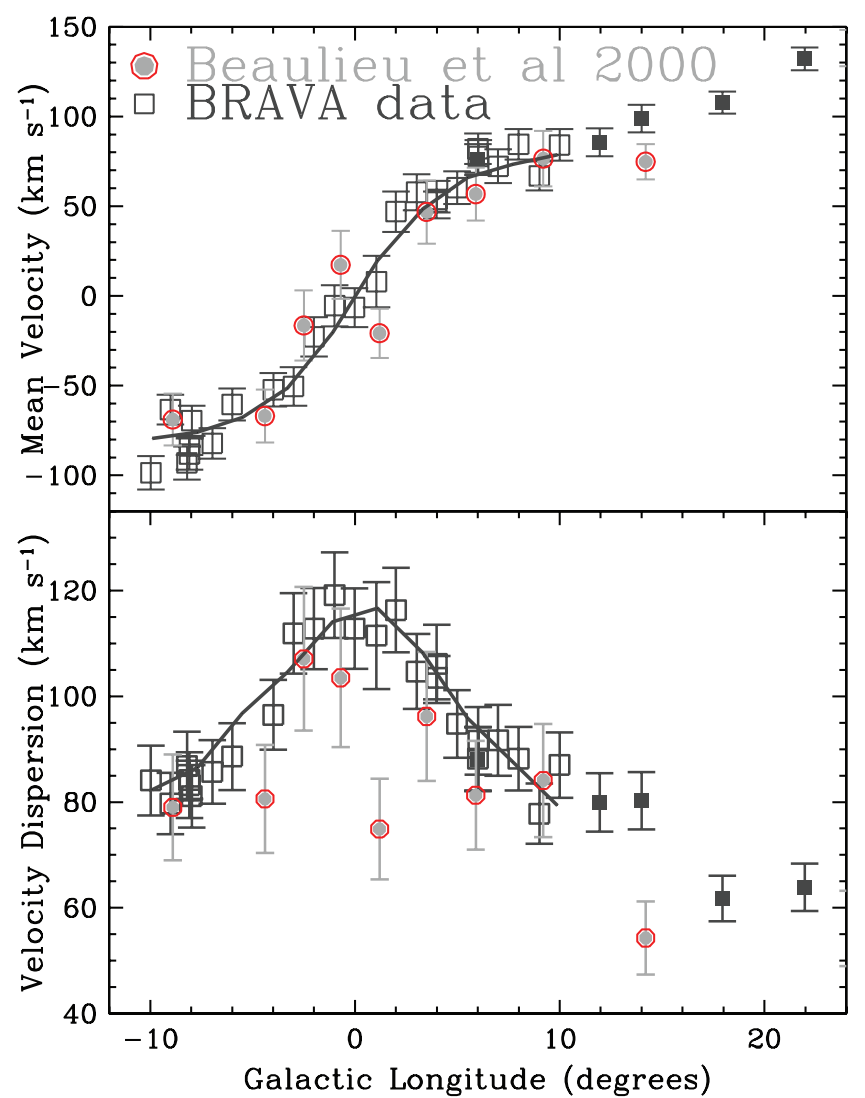

Figure 12. Velocity dispersion profile (bottom) and rotation curve (top) for all the $b=-4^{\circ}$ fields. The open symbols indicate data already published and the filled symbols indicate the data presented here. Overplotted is data from bulge PNe from Beaulieu et al. (2000), as well as the predictions of the Shen et al. (2010) model.

(A color version of this figure is available in the online journal.)

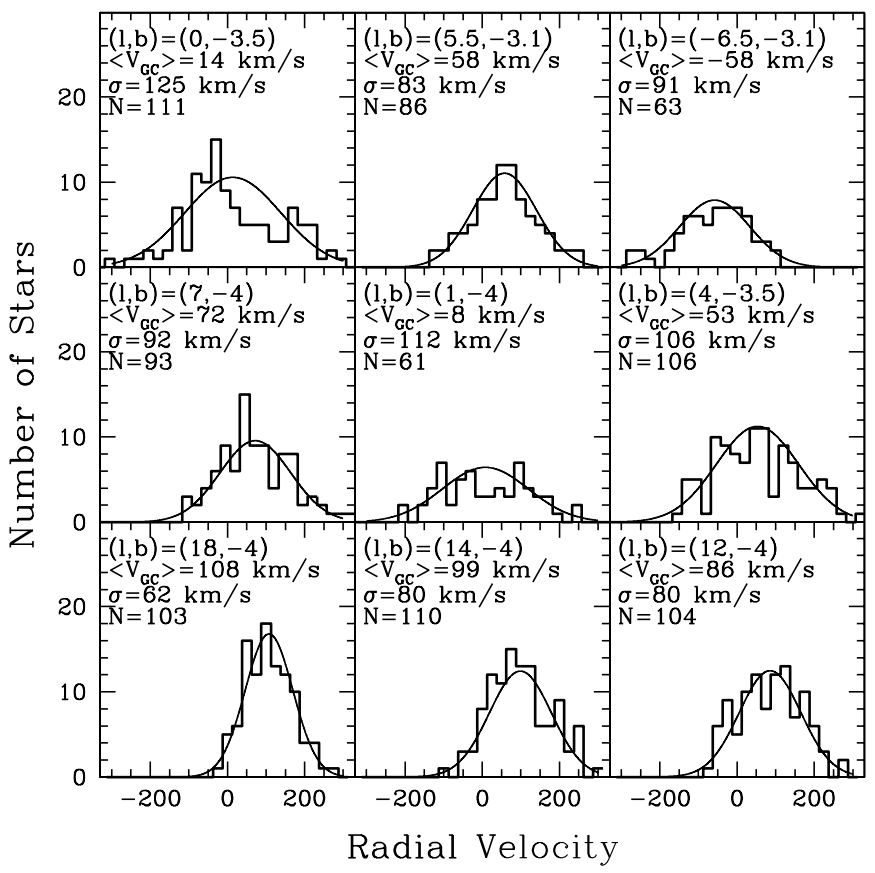

Figure 13. Presentation of all bulge field galactocentric velocity distributions. Overlaid on each plot is a Gaussian derived from the field statistics. 

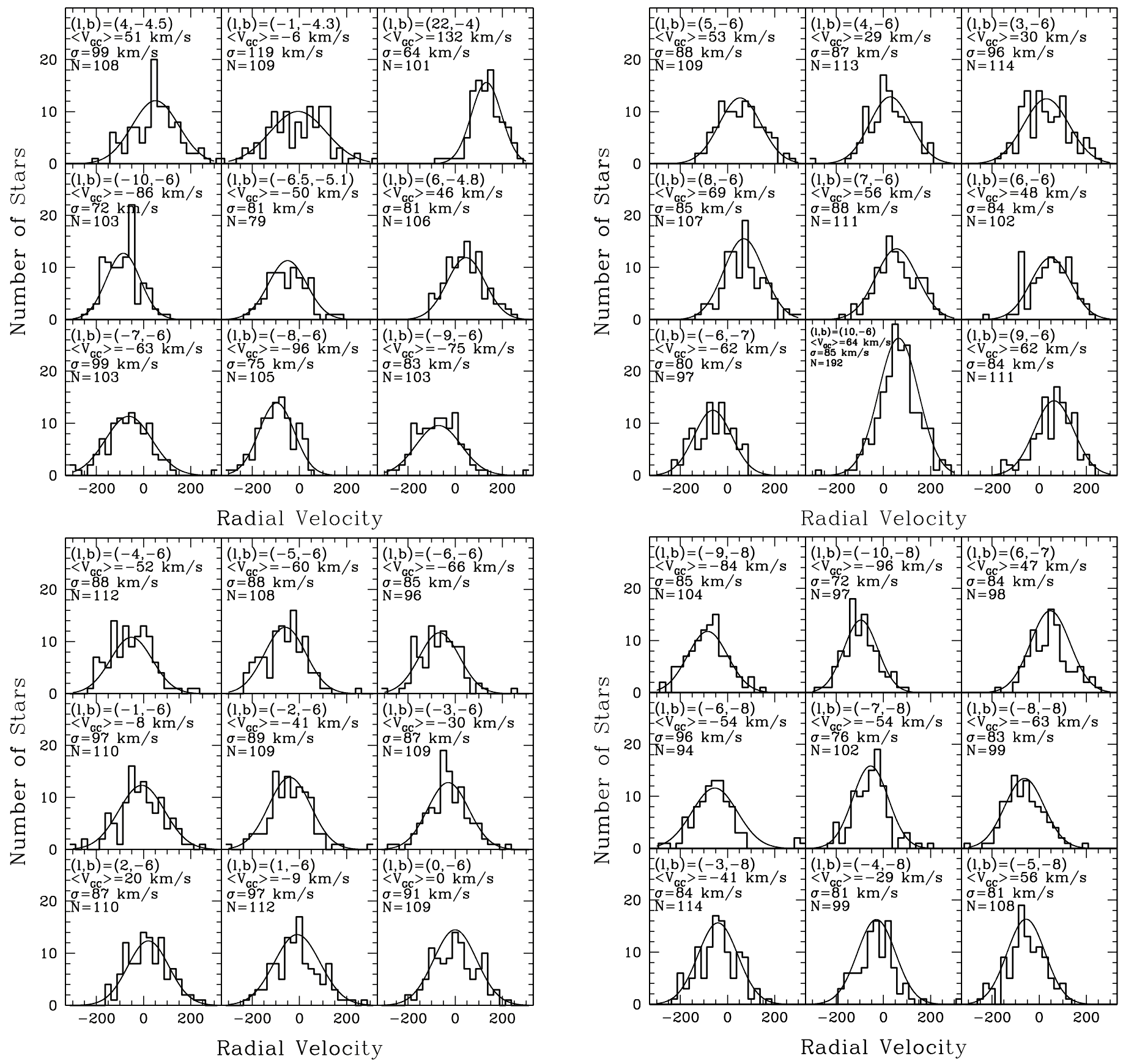

Figure 13. (Continued)

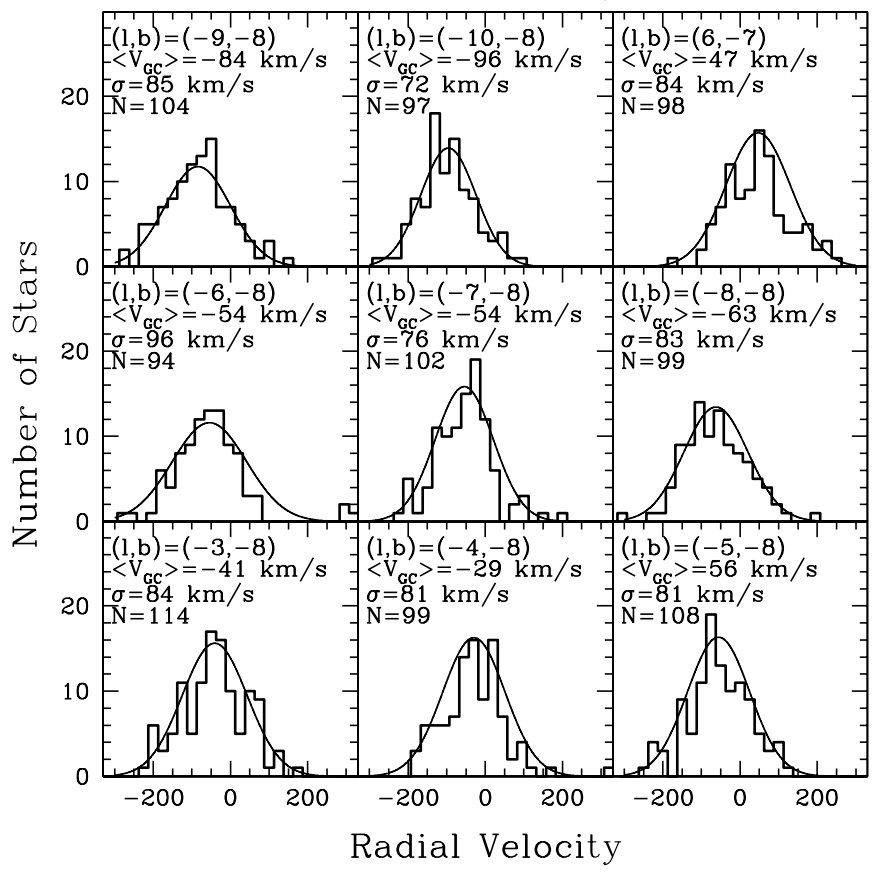

Figure 13. (Continued)

each field $(\sim 100)$, deviations from a normal distribution are not unexpected in random draws. However, fields with the largest deviations from a normal distribution may aid in the selection of follow-up observations. To test for normality, the Shapiro-Wilk test (Shapiro \& Wilk 1965; D’Agostino 1986) and the Anderson-Darling test (Press et al. 1986) are employed. These normality tests are two of the most powerful tests for deviations from normality and are especially useful for sample sizes that are relatively small, i.e., $\sim 100$ particles. For example, the Shapiro-Wilk normality test has successfully been used by Kepley et al. (2007) to detect known streams in one component of velocity. For both of these tests, the smaller the $p$-value, the less likely it is that the data come from a normal distribution, and a $p$-value $<0.05$ rejects the supposition of normality. The results of these tests are given in Table 3. The tested BRAVA field is first listed in Table 3 followed by the number of stars in the field, the skewness and kurtosis, and its associated $p$-value from the Shapiro-Wilk test and from the Anderson-Darling test.

In general, the skewness and kurtosis values are all within $1 \sigma$ or $2 \sigma$, the exception being fields $(l, b)=(-1,-8),(-4,-8)$, $(-6,-8),(8,-8)$, and $(9,-8)$. Upon closer examination, it is apparent that except for $(l, b)=(-6,-8)$, which is discussed in more detail below, these fields each contain a star on the tail end of the distribution which greatly influences the skewness and kurtosis values. All of these fields also fail the Shapiro-Wilk normality test, which gives more weight to the tails of the distribution than the Anderson-Darling test. All of these fields are located at $b=-8^{\circ}$, and it is likely that we are seeing some signatures of the bulge/disk boundary in these fields. 

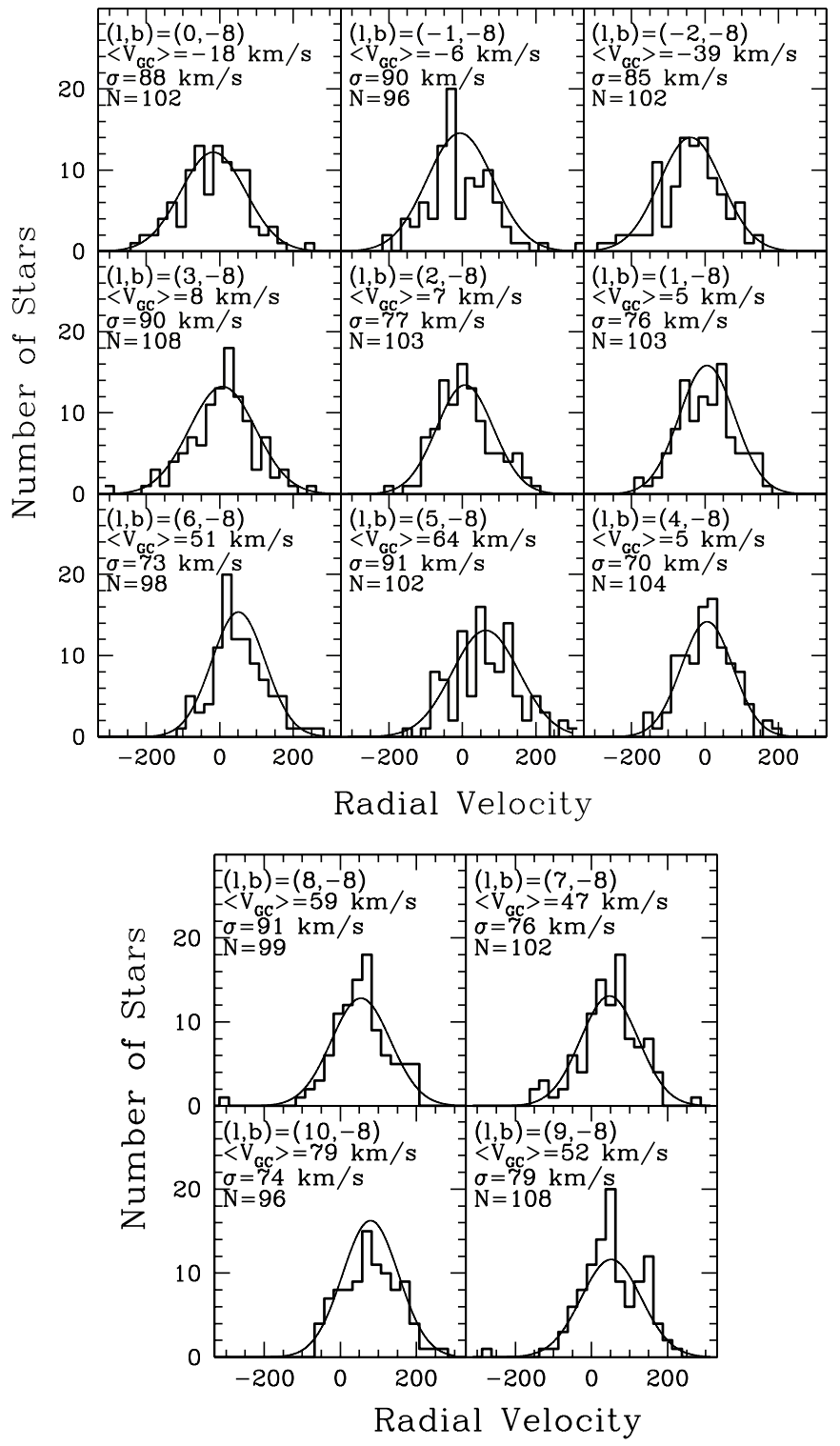

Figure 13. (Continued)

There are only two BRAVA fields that fail both the Shapiro-Wilk and the Anderson-Darling test. These fields are located at $(l, b)=(0,-3.5)$ and $(l, b)=(-6,-8)$. The former has a skewness and kurtosis consistent with a Gaussian distribution, but $p$-values that reject the supposition of normality. Removing the stars that lie in the tails of the distribution does not cause the $p$-value to increase and hence indicate normality. There is a strong peak in the velocity distribution at around $-30 \mathrm{~km} \mathrm{~s}^{-1}$ and there are not many stars at $\sim 100 \mathrm{~km} \mathrm{~s}^{-1}$, which are the likely features causing the Shapiro-Wilk and Anderson-Darling tests to reject normality. Interestingly, the possible signature of a disrupted satellite reported by Rangwala \& Williams (2009) at $(l, b)=(+5.5,-3.5)$ has a velocity distribution excess around $-35 \mathrm{~km} \mathrm{~s}^{-1}$. However, we find no correlation at $(l, b)=(0,-3.5)$ with the stars that have velocities around $-30 \mathrm{~km} \mathrm{~s}^{-1}$ and their $\mathrm{TiO}$ values. Our sample size is small, and follow-up observations of this field would be particularly interesting.

The field at $(l, b)=(-6,-8)$, on the other hand, has the largest skewness and kurtosis of any BRAVA fields (1.38 \pm 0.25 and $4.91 \pm 0.51$, respectively). Furthermore, it has three stars with heliocentric velocities, $\mathrm{V}_{\mathrm{HC}}$, greater than $300 \mathrm{~km} \mathrm{~s}^{-1}$; the radial
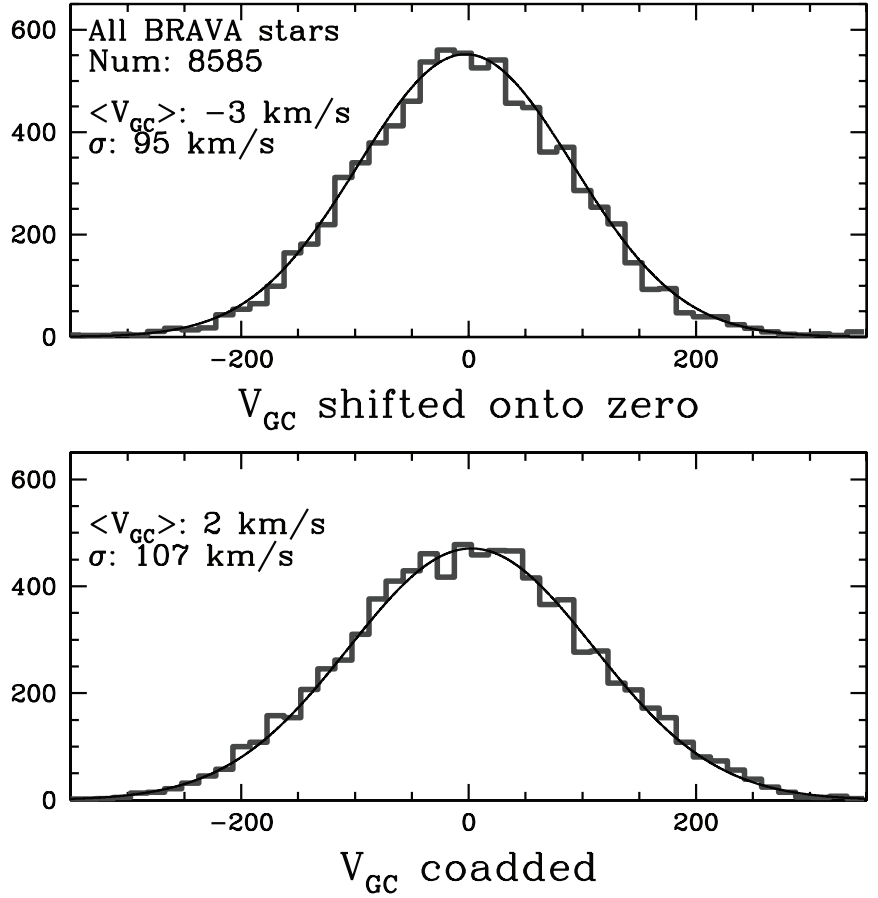

Figure 14. Co-added galactocentric radial velocities of the BRAVA stars. In the top panel, each BRAVA field is shifted to zero before co-addition. A bin width of $15 \mathrm{~km} \mathrm{~s}^{-1}$ is used for both histograms. Neither distribution shows any signs of deviation from a Gaussian.

velocity dispersion of these three stars is $16 \pm 7 \mathrm{~km} \mathrm{~s}^{-1}$. From our complete sample of 8585 stars, only 11 stars have velocities that are above $300 \mathrm{~km} \mathrm{~s}^{-1}$, and so it is striking that 3 of these stars are located in this field. Again it would be especially interesting to obtain follow-up observations of the stars in this field to see if more stars with such large velocities are found.

Figure 14 shows all the 8585 radial velocities obtained from the BRAVA data set co-added (bottom panel). The mean is $2 \pm 1 \mathrm{~km} \mathrm{~s}^{-1}$ with a $\sigma=107 \pm 1 \mathrm{~km} \mathrm{~s}^{-1}$. The skewness is negligible $(0.03 \pm 0.03)$ and the kurtosis of $-0.17 \pm 0.05$ implies a slightly platykurtic (flattened) distribution. Both of these results are in agreement with the results found in Paper I. Furthermore, the lack of significant skewness and a small value of kurtosis is consistent with our argument that our bulge radial velocity distribution is not largely contaminated by either cold components (disk) or hot components (halo).

A Shapiro-Wilk normality test fails for this co-added data set because the sample is too large; with 8585 stars, there are many radial velocities with very similar values. An Anderson-Darling normality test gives a $p$-value of 0.59 and a Kolmogorov-Smirnov test, which works best with large data sets, gives a $p$-value of 0.10 . These results suggest no significant deviations from a Gaussian distribution.

The top panel of Figure 14 shows the radial velocities shifted onto zero and then co-added. The mean is $-3 \pm 1 \mathrm{~km} \mathrm{~s}^{-1}$ with a $\sigma=95 \pm 1 \mathrm{~km} \mathrm{~s}^{-1}$. Again, the distribution is Gaussian, with no apparent deviation from a normal distribution. It is noteworthy that there is only one star in our sample with a velocity greater than $\pm 4 \sigma$, which is discussed in Section 4.

Figure 15 shows the longitude-velocity plot for the three BRAVA major-axis strips. For latitudes closest to the plane $(b=$ $-4^{\circ}$ and $b=-6^{\circ}$ ) there is no evidence of a cold disk component in our sample which would manifest itself as a linear trend. However, farther from the plane $\left(b=-8^{\circ}, \sim 1.2 \mathrm{kpc}\right.$ from the 
Table 3

BRAVA Rotation and Dispersion Results

\begin{tabular}{|c|c|c|c|c|c|c|c|c|}
\hline $\begin{array}{l}\text { gal } l \\
\text { (deg) }\end{array}$ & $\begin{array}{l}\text { gal } b \\
\text { (deg) }\end{array}$ & Number Velocities & Skewness & $\sigma_{\text {Skew }}$ & Kurtosis & $\sigma_{\text {kurt }}$ & $\begin{array}{c}p \text {-index } \\
\text { (Shapiro-Wilk) }\end{array}$ & $\begin{array}{c}p \text {-index } \\
\text { (Anderson-Darling) }\end{array}$ \\
\hline-6.5 & -3.1 & 63 & -0.60 & 0.31 & 0.07 & 0.62 & 0.043 & 0.126 \\
\hline 5.5 & -3.1 & 86 & 0.05 & 0.26 & -0.31 & 0.53 & 0.842 & 0.991 \\
\hline 0.0 & -3.5 & 111 & 0.19 & 0.23 & -0.41 & 0.46 & 0.035 & 0.004 \\
\hline 4.0 & -3.5 & 106 & 0.22 & 0.24 & -0.64 & 0.48 & 0.182 & 0.266 \\
\hline 1.0 & -4.0 & 61 & 0.14 & 0.31 & 0.86 & 0.63 & 0.238 & 0.267 \\
\hline 7.0 & -4.0 & 93 & -0.08 & 0.25 & 0.37 & 0.51 & 0.386 & 0.502 \\
\hline 6.0 & -4.0 & 106 & -0.11 & 0.24 & 0.09 & 0.48 & 0.967 & 0.943 \\
\hline 12.0 & -4.0 & 104 & 0.07 & 0.24 & -0.71 & 0.48 & 0.203 & 0.412 \\
\hline 14.0 & -4.0 & 110 & 0.28 & 0.23 & -0.23 & 0.47 & 0.500 & 0.306 \\
\hline 18.0 & -4.0 & 103 & 0.27 & 0.24 & -0.38 & 0.48 & 0.434 & 0.498 \\
\hline 22.0 & -4.0 & 101 & -0.38 & 0.24 & 0.72 & 0.49 & 0.258 & 0.567 \\
\hline-1.0 & -4.3 & 109 & -0.17 & 0.23 & -0.09 & 0.47 & 0.513 & 0.160 \\
\hline 4.0 & -4.5 & 108 & -0.05 & 0.24 & 0.02 & 0.47 & 0.750 & 0.384 \\
\hline 6.0 & -4.8 & 106 & 0.26 & 0.24 & -0.37 & 0.48 & 0.357 & 0.747 \\
\hline-6.5 & -5.1 & 79 & 0.22 & 0.28 & -0.06 & 0.55 & 0.789 & 0.728 \\
\hline-10.0 & -6.0 & 103 & 0.15 & 0.24 & -0.36 & 0.48 & 0.440 & 0.163 \\
\hline-9.0 & -6.0 & 102 & -0.02 & 0.24 & -0.50 & 0.49 & 0.899 & 0.883 \\
\hline-8.0 & -6.0 & 105 & -0.24 & 0.24 & -0.26 & 0.48 & 0.449 & 0.762 \\
\hline-7.0 & -6.0 & 103 & -0.04 & 0.24 & 1.96 & 0.48 & 0.087 & 0.495 \\
\hline-6.0 & -6.0 & 96 & 0.43 & 0.25 & 1.43 & 0.50 & 0.116 & 0.542 \\
\hline-5.0 & -6.0 & 108 & 0.25 & 0.24 & 0.57 & 0.47 & 0.075 & 0.329 \\
\hline-4.0 & -6.0 & 112 & 0.38 & 0.23 & 0.11 & 0.46 & 0.084 & 0.426 \\
\hline-3.0 & -6.0 & 109 & 0.01 & 0.23 & 0.55 & 0.47 & 0.803 & 0.506 \\
\hline-2.0 & -6.0 & 109 & 0.06 & 0.23 & 1.62 & 0.47 & 0.130 & 0.364 \\
\hline-1.0 & -6.0 & 110 & -0.18 & 0.23 & 0.31 & 0.47 & 0.898 & 0.835 \\
\hline 0.0 & -6.0 & 109 & 0.09 & 0.23 & -0.62 & 0.47 & 0.360 & 0.298 \\
\hline 1.0 & -6.0 & 112 & 0.22 & 0.23 & 0.03 & 0.46 & 0.793 & 0.614 \\
\hline 2.0 & -6.0 & 110 & 0.26 & 0.23 & -0.36 & 0.47 & 0.517 & 0.754 \\
\hline 3.0 & -6.0 & 114 & 0.24 & 0.23 & -0.55 & 0.46 & 0.092 & 0.109 \\
\hline 4.0 & -6.0 & 113 & -0.33 & 0.23 & 0.60 & 0.46 & 0.083 & 0.556 \\
\hline 5.0 & -6.0 & 109 & 0.18 & 0.23 & -0.58 & 0.47 & 0.436 & 0.523 \\
\hline 6.0 & -6.0 & 102 & -0.35 & 0.24 & -0.09 & 0.49 & 0.104 & 0.121 \\
\hline 7.0 & -6.0 & 111 & -0.03 & 0.23 & -0.37 & 0.46 & 0.661 & 0.507 \\
\hline 8.0 & -6.0 & 107 & 0.31 & 0.24 & 0.14 & 0.47 & 0.572 & 0.459 \\
\hline 9.0 & -6.0 & 111 & -0.48 & 0.23 & 0.03 & 0.46 & 0.680 & 0.186 \\
\hline 10.0 & -6.0 & 192 & -0.19 & 0.18 & 0.79 & 0.35 & 0.153 & 0.342 \\
\hline-6.0 & -6.9 & 97 & -0.12 & 0.25 & -0.80 & 0.50 & 0.121 & 0.292 \\
\hline 6.0 & -6.9 & 98 & 0.32 & 0.25 & -0.22 & 0.49 & 0.133 & 0.110 \\
\hline-10.0 & -8.0 & 97 & 0.27 & 0.25 & 0.07 & 0.50 & 0.622 & 0.443 \\
\hline-9.0 & -8.0 & 104 & 0.00 & 0.24 & -0.01 & 0.48 & 0.894 & 0.864 \\
\hline-8.0 & -8.0 & 99 & 0.24 & 0.25 & 0.53 & 0.49 & 0.655 & 0.545 \\
\hline-7.0 & -8.0 & 102 & 0.41 & 0.24 & 1.01 & 0.49 & 0.059 & 0.117 \\
\hline-6.0 & -8.0 & 94 & 1.38 & 0.25 & 4.91 & 0.51 & 0.0001 & 0.0001 \\
\hline-5.0 & -8.0 & 108 & -0.06 & 0.24 & -0.10 & 0.47 & 0.627 & 0.649 \\
\hline-4.0 & -8.0 & 99 & 0.78 & 0.25 & 2.68 & 0.49 & 0.002 & 0.227 \\
\hline-3.0 & -8.0 & 114 & 0.01 & 0.23 & -0.32 & 0.46 & 0.466 & 0.509 \\
\hline-2.0 & -8.0 & 102 & -0.42 & 0.24 & 0.16 & 0.49 & 0.255 & 0.320 \\
\hline-1.0 & -8.0 & 96 & 0.71 & 0.25 & 2.42 & 0.50 & 0.006 & 0.079 \\
\hline 0.0 & -8.0 & 102 & -0.01 & 0.24 & 0.03 & 0.49 & 0.922 & 0.773 \\
\hline 1.0 & -8.0 & 103 & -0.05 & 0.24 & 0.40 & 0.48 & 0.762 & 0.686 \\
\hline 2.0 & -8.0 & 103 & 0.21 & 0.24 & -0.09 & 0.48 & 0.467 & 0.268 \\
\hline 3.0 & -8.0 & 108 & -0.33 & 0.24 & 0.89 & 0.47 & 0.422 & 0.299 \\
\hline 4.0 & -8.0 & 104 & 0.11 & 0.24 & -0.13 & 0.48 & 0.802 & 0.934 \\
\hline 5.0 & -8.0 & 102 & 0.24 & 0.24 & -0.05 & 0.49 & 0.519 & 0.481 \\
\hline 6.0 & -8.0 & 98 & 0.43 & 0.25 & 0.29 & 0.49 & 0.173 & 0.110 \\
\hline 7.0 & -8.0 & 102 & -0.23 & 0.24 & 0.60 & 0.49 & 0.169 & 0.182 \\
\hline 8.0 & -8.0 & 99 & -0.89 & 0.25 & 4.16 & 0.49 & 0.0001 & 0.090 \\
\hline 9.0 & -8.0 & 108 & -0.61 & 0.24 & 1.89 & 0.47 & 0.007 & 0.288 \\
\hline 10.0 & -8.0 & 96 & 0.06 & 0.25 & -0.61 & 0.50 & 0.460 & 0.585 \\
\hline
\end{tabular}




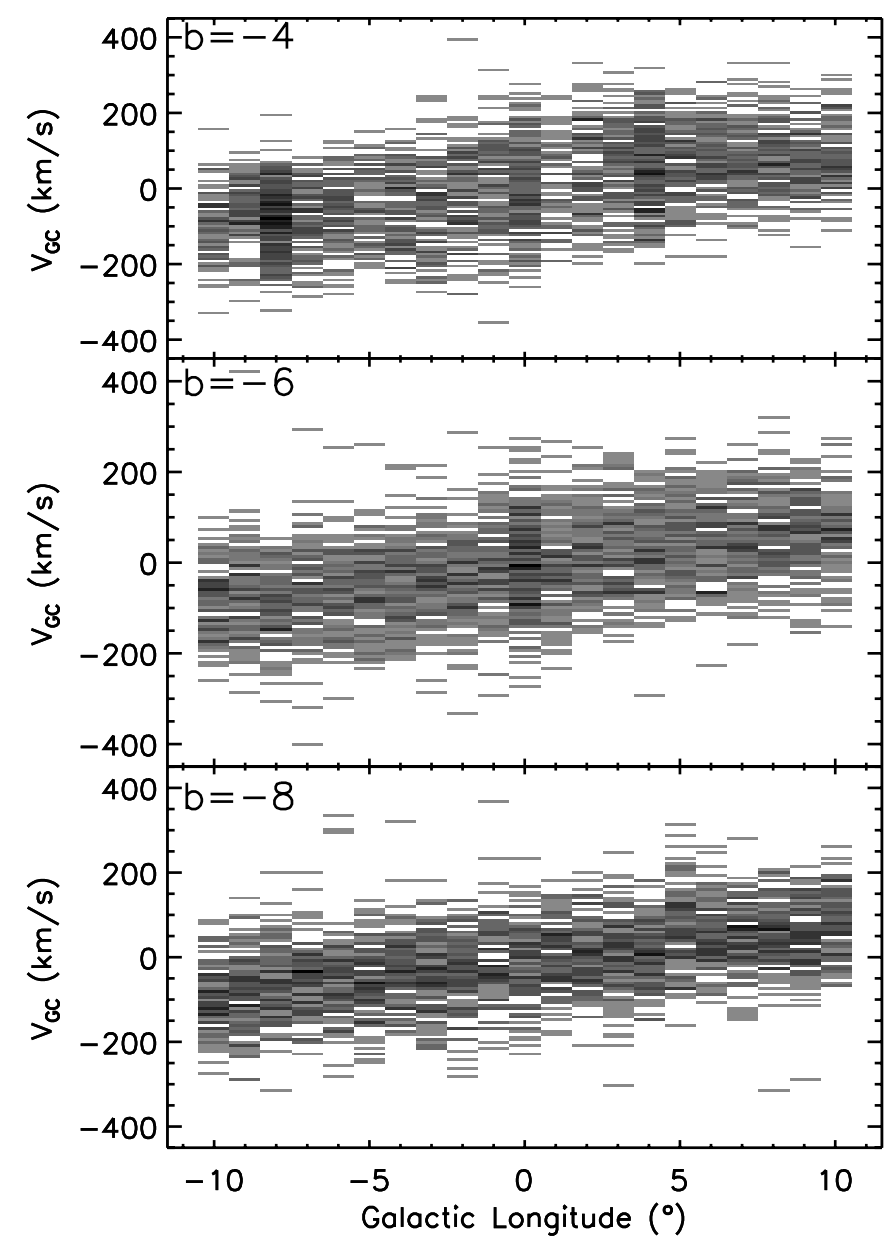

Figure 15. Longitude-velocity $\left(l_{v}\right)$ plot for the entire bulge sample at $b=-4^{\circ}$ (top), $b=-6^{\circ}$ (middle), and $b=-8^{\circ}$ (bottom). The $l_{v}$ plot is smoothed to $1^{\circ}$ in longitude and $10 \mathrm{~km} \mathrm{~s}^{-1}$ in galactocentric velocity. This figure shows the cylindrical rotation trend very clearly. Note the lack of any prominent "cold" features that would indicate a possible stream detection across multiple fields.

Galactic plane), the " $S$ " shape is not as apparent, suggesting that the disk does contribute to the BRAVA sample in this regime. Indeed, as compared to the $b=-4^{\circ}$ and $b=-6^{\circ}$ fields, the $b=-8^{\circ}$ fields are also those that in general have skewness, kurtosis, and $p$-values that are least consistent with a normal distribution (see Table 3). Recent studies of PNe in the inner Milky Way suggest a bulge-disk interface at $\sim 1.5 \mathrm{kpc}$ (Cavichia et al. 2011), consistent with our findings.

Figure 16 shows the latitude-velocity plot for three BRAVA minor axis strips. As expected a linear trend is seen; neither cold components nor indications of a hot, non-rotating population are seen. However, we see "rotation" in the sense of solid body rotation.

\subsection{A Milky Way Pseudobulge?}

A vertical metallicity gradient in the bulge has been reported in several recent studies (Meléndez et al. 2008; Zoccali et al. 2008; Babusiaux et al. 2010; Johnson et al. 2011), and it has often been used as an argument against the boxy bulge/bar model of Shen et al. (2010). It is true that the collective bar buckling happens to essentially the whole disk that is in place at the time of the buckling instability, but a vertical metallicity gradient could still be consistent with the Shen bar/pseudobulge model. One possibility is that some of the vertical thickening could be produced by resonant heating of stars that scatter off the bar

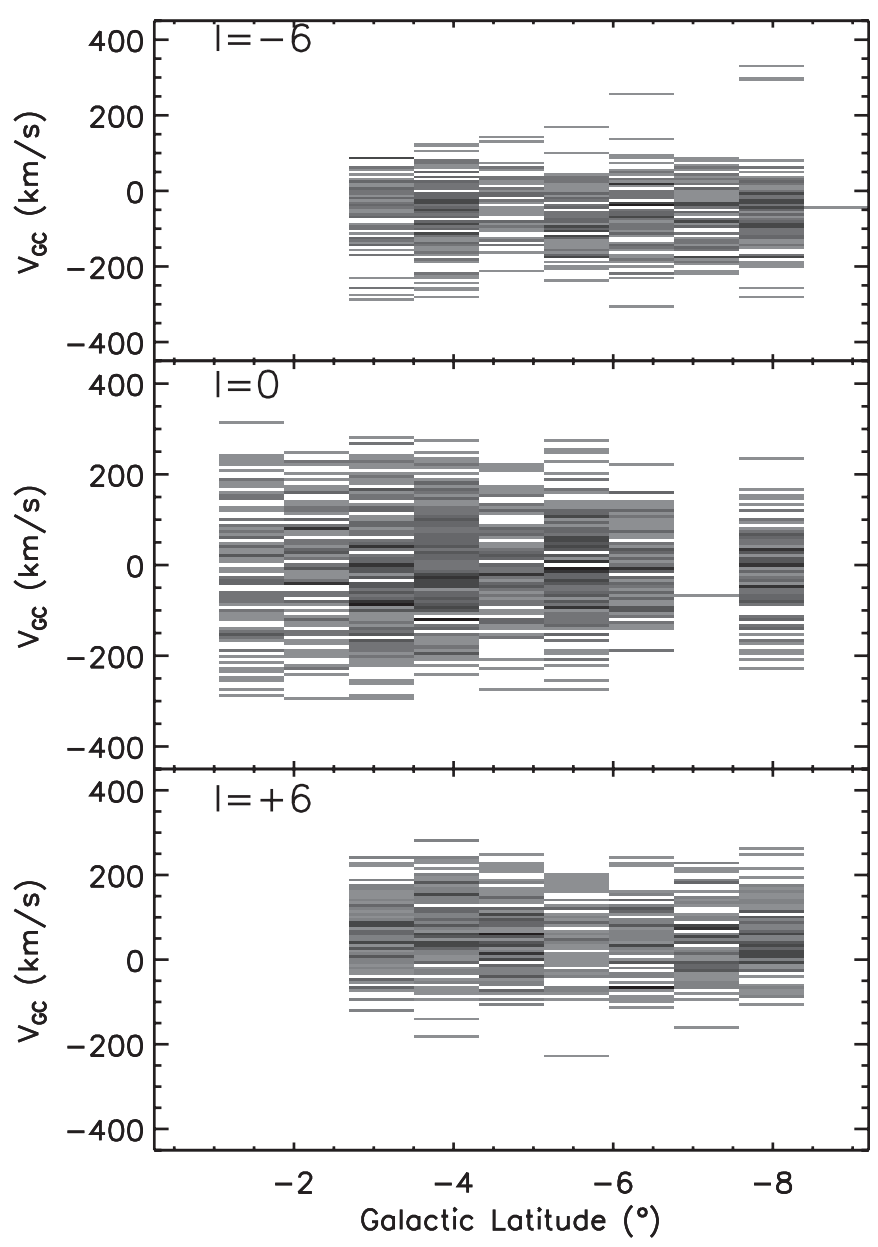

Figure 16. Latitude-velocity $\left(b_{v}\right)$ plot for the entire bulge sample at $l=-6^{\circ}$ (top), $l=0^{\circ}$ (middle), and $b=+6^{\circ}$ (bottom). The sample is smoothed to $1^{\circ}$ in latitude.

(Pfenniger 1984, 1985; Pfenniger \& Norman 1990). If the most metal-poor stars are also the oldest stars, then they have been scattered for the longest time and now reach the greatest heights, hence giving rise to a vertical metallicity gradient. Second, the Shen et al. (2010) model still allows a relatively small mergerbuilt classical bulge (about $10 \%$ of the disk mass); the mixing of two bulge populations could conceivably produce a vertical metallicity gradient (also see the Besancon model; Robin et al. 2011). A mixing of the bar/pseudobulge with the inner thick disk was also proposed to explain the vertical metallicity gradient (Bekki \& Tsujimoto 2011).

That the Milky Way has a pseudobulge is consistent with the majority of the bright galaxies in the local $11 \mathrm{Mpc}$ volume (Kormendy et al. 2010; Fisher \& Drory 2011), especially for those galaxies with a mass similar to that of the Milky Way. Recently, an in-depth study of NGC 4565 has revealed that this galaxy also contains a pseudobulge and no hint of a mergerbuilt classical bulge (Kormendy \& Barentine 2010). Like the Milky Way, NGC 4565 has a peanut-shaped bulge which rotates cylindrically (Kormendy \& Illingworth 1982) and has a strong abundance gradient (Proctor et al. 2000). Hence other galaxies besides the Milky Way with an abundance gradient but no (or very little) classical bulge are known to exist. Evolution of the vertical metallicity gradient, however, has not been extensively investigated by theoretical studies, but the model by Bekki \& Tsujimoto (2011) is a step forward in understanding why an 
abundance gradient is observed in the bulge even if the bar evolved secularly.

\section{CONCLUSIONS}

We have presented the final data set of the BRAVA survey and provided a Web site for data access, which is available at the IRSA archive: http://irsa.ipac.caltech.edu/ as well as at UCLA: http://brava.astro.ucla.edu/. The observations at the $b=$ $-6^{\circ}$ latitude fields as well as more observations at the $b=-8^{\circ}$ latitude fields confirm previous suggestions that the Galactic bulge rotates cylindrically, as do boxy bulges of other galaxies (e.g., Kormendy \& Illingworth 1982; Jarvis 1990; Shaw 1993; Kormendy \& Kennicutt 2004; Howard et al. 2009). The data in this paper double the number of stars shown in Paper I and Howard et al. (2009), and used in Shen et al. (2010). Analysis of these data in the same fashion shows that the Galactic bulge consists of a single massive bar formed by secular evolution. The existence of such a massive bar and no classical bulge implies that our Galaxy has not suffered any large mergers (enough to form a classical bulge) since the epoch at which the disk formed. Based on $\mathrm{TiO} \varepsilon$ index measurements we qualitatively see the signatures of a vertical metallicity gradient-an attribute that is in contrast to our kinematic characterization of the bulge as a pure pseudobulge. A detailed analysis of the BRAVA metallicity scale and its spatial distribution is clearly warranted and underway.

It is our pleasure to thank Inma Martinez-Valpuesta and Ortwin Gerhard for helpful discussions. A. Kunder, R. M. Rich, C. I. Johnson, J. Shen, A. C. Robin, M. Soto, P. Frinchaboy, Y. Wang, and L. Origlia wish to acknowledge the hospitality of the Aspen Center for Physics, which is supported by the NSF Grant 1066293, and where much dialogue and exchange of ideas took place. A. Kunder thanks Dan Phillips for his work on the BRAVA Web site. A. Koch thanks the Deutsche Forschungsgemeinschaft for funding from Emmy-Noether grant Ko 4161/1. This material is based upon work supported by the National Science Foundation under award AST-0709479 to R. M. Rich and award AST-1003201 to C.I.J. The research presented here is partially supported by the National Natural Science Foundation of China under grant no. 11073037 to J. Shen and by 973 Program of China under grant no. 2009CB824800 to J. Shen. We thank the anonymous referee for suggestions that strengthened our analysis.

\section{REFERENCES}

Babusiaux, C., Gómez, A., Hill, V., et al. 2010, A\&A, 519, 77

Beaulieu, S. F., Freeman, K. C., Kalnajs, A. J., Saha, P., \& Zhao, H. 2000, AJ, 120,855

Bekki, K., \& Tsujimoto, T. 2011, MNRAS, 416, 60

Bissantz, N., \& Gerhard, O. 2002, MNRAS, 330, 59

Blanco, V. M., McCarthy, M. F., \& Blanco, B. M. 1984, AJ, 89, 636

Blitz, L., \& Spergel, D. N. 1991, ApJ, 370, 205
Brown, W. R., Geller, M. J., Kenyon, S. J., \& Kurtz, M. J. 2005, ApJ, 622, L33 Cabrera-Lavers, A., Hammersley, P. L., González-Fernández, C., et al. 2007, A\&A, 465, 825

Cavichia, O., Costa, R. D. D., \& Maciel, W. J. 2011, RevMexAA, 47, 49

D'Agostino, R. B. 1986, in Goodness-of-Fit Techniques, ed. R. B. D'Agostino \& M. A. Stephens (New York: Dekker), 367

De Propris, R., Rich, R. M., Kunder, A., et al. 2011, ApJ, 732, L36

Fisher, D. B., \& Drory, N. 2011, ApJ, 733, L47

Frogel, J. A., Tiede, G. P., \& Kuchinski, L. E. 1999, AJ, 117, 2296

Frogel, J. A., \& Whitford, A. E. 1987, ApJ, 320, L199

Fulbright, J. P., McWilliam, A., \& Rich, R. M. 2006, ApJ, 636, 821

Gonzalez, O. A., Rejkuba, M., Zoccali, M., et al. 2011, A\&A, 530, 54

Hammersley, P. L., Garzón, F., Mahoney, T. J., López-Corredoira, M., \& Torres, M. A. P. 2000, MNRAS, 317, L45

Hauschildt, P. H., Allard, F., \& Baron, E. 1999, ApJ, 512, 377

Hauschildt, P. H., Allard, F., Baron, E., Aufdenberg, J., \& Schweitzer, A. 2003, in ASP Conf. Ser. 298, GAIA Spectroscopy: Science and Technology, ed U. Munari (San Francisco, CA: ASP), 179

Howard, C. D., Rich, R. M., Clarkson, W., et al. 2009, ApJ, 702, L153

Howard, C. D., Rich, R. M., Reitzel, D. B., et al. 2008, ApJ, 688, 1060

Jarvis, B. 1990, in Dynamics and Interactions of Galaxies, ed. R. Wielen (New York: Springer), 416

Johnson, C. I., Rich, R. M., Fulbright, J. P., Valenti, E., \& McWilliam, A. 2011, ApJ, 732, 108

Kenyon, S. J., Bromley, B. C., Geller, M. J., \& Brown, W. R. 2008, ApJ, 680, 312

Kepley, A. A., Morrison, H. L., Helmi, A., et al. 2007, AJ, 134, 1579

Kormendy, J., \& Barentine, J. C. 2010, ApJ, 715, L176

Kormendy, J., Drory, N., Bender, R., \& Cornell, M. E. 2010, ApJ, 723, 54

Kormendy, J., \& Illingworth, G. 1982, ApJ, 256, 460

Kormendy, J., \& Kennicutt, R. C. 2004, ARA\&A, 42, 603

Launhardt, R., Zylka, R., \& Mezger, P. G. 2002, A\&A, 384, 112

Liszt, H. S., \& Burton, M. B. 1980, ApJ, 236, 779

Meléndez, J., Asplund, M., Alves-Brito, A., et al. 2008, A\&A, 484, L21

Milone, A., \& Barbuy, B. 1994, A\&AS, 108, 449

Mould, J. R. 1986, in Stellar Populations, ed. A. Renzini \& M. Tosi (Cambridge: Cambridge Univ. Press), 9

Nassau, J. J., \& Blanco, V. M. 1958, ApJ, 128, 46

Pfenniger, D. 1984, A\&A, 134, 373

Pfenniger, D. 1985, A\&A, 150, 112

Pfenniger, D., \& Norman, C. 1990, ApJ, 363, 391

Press, W. H., Flannery, B. P., \& Teukolsky, S. A. 1986, Numerical Recipes, The Art of Scientific Computing (Cambridge: Cambridge Univ. Press)

Proctor, R. N., Sansom, A. E., \& Reid, I. N. 2000, MNRAS, 311, 37

Rangwala, N., \& Williams, T. B. 2009, ApJ, 702, 414

Reitzel, D. B., Koch, A., Rich, R. M., et al. 2007, BAAS, 39, 897

Rich, R. M., \& Origlia, L. 2005, ApJ, 634, 1293

Rich, R. M., Origlia, L., \& Valenti, E. 2007a, ApJ, 665, L119

Rich, R. M., Reitzel, D. B., Howard, C. D., \& Zhao, H. 2007b, ApJ, 658, L29

Robin, A. C., Marshall, D. J., Schultheis, M., et al. 2011, A\&A, in press (arXiv:1111.5744)

Rutledge, G. A., Hesser, J. E., Stetson, P. B., et al. 1997, PASP, 109, 883

Schlegel, D. J., Finkbeiner, D. P., \& Davis, M. 1998, ApJ, 500, 525

Sevenster, M., Saha, P., Valls-Gabaud, D., \& Fux, R. 1999, MNRAS, 307, 584

Shapiro, S. S., \& Wilk, M. B. 1965, Biometrika, 52, 591

Sharples, R., Walker, A., \& Cropper, M. 1990, MNRAS, 246, 54

Shaw, M. 1993, A\&A, 280, 33

Shen, J., Rich, M. R., Kormendy, J., et al. 2010, ApJ, 720, L72

Skrutskie, M. F., Cutri, R. M., Stiening, R., et al. 2006, AJ, 131, 1163

Sumi, T. 2004, MNRAS, 348, 1439

van Dokkum, P. G. 2001, PASP, 113, 1420

Zhao, H. 1996, in ASP Conf. Ser. 91, Barred Galaxies, ed. R. Buta, D. A Crocker, \& B. G. Elmegreen (San Francisco, CA: ASP), 549

Zoccali, M., Hill, V., Lecureur, A., et al. 2008, A\&A, 486, 177 